RI-8-95

hep-th/9509013

September 1995

\title{
WZNW Models and Gauged WZNW Models Based On a Family of Solvable Lie Algebras
}

\author{
Amit Giveon円, Oskar Pelc' and Eliezer Rabinovici户 \\ Racah Institute of Physics, The Hebrew University \\ Jerusalem, 91904, Israel
}

\begin{abstract}
A family of solvable self-dual Lie algebras that are not double extensions of Abelian algebras and, therefore, cannot be obtained through a Wigner contraction, is presented. We construct WZNW and gauged WZNW models based on the first two algebras in this family. We also analyze some general phenomena arising in such models.
\end{abstract}

\footnotetext{
${ }^{1}$ E-mail: giveon@vms.huji.ac.il

${ }^{2}$ E-mail: oskar@shum.cc.huji.ac.il

${ }^{3}$ E-mail: eliezer@vms.huji.ac.il
} 


\section{Introduction}

WZNW models [1] and gauged WZNW models [2] have served as building blocks of various string theories. For the construction of a WZNW model, one needs a Lie group, and also a metric - a non-degenerate, symmetric bilinear form - on the corresponding Lie algebra. A Lie algebra that admits such a metric is called self-dual.

At first reductive algebras (direct sums of semi-simple and Abelian algebras) were considered. Such algebras have natural candidates for the invariant metric - the Killing form for the semi-simple part and an arbitrary metric for the Abelian part. However, there exist self-dual algebras that are not reductive and these also can be used for the construction of WZNW models (and their supersymmetric extensions) [3]-14.

One of the interesting features of an affine Sugawara construction based on a non-semisimple (and indecomposable - not an orthogonal direst sum) algebra is that the resulting central charge is integer and equals to the dimension of the algebra [5] 15]. This may be a sign of some interesting phenomena. When the algebra can be obtained through a Wigner contraction [16] of a semi-simple algebra, as described in [5], this is explained by the fact that in the contraction process, a semi-classical limit is taken - the levels of the simple components are taken to infinity. This suggests that the resulting model has a free-field representation (this was demonstrated for the example of [3] in [ [4]).

In this paper we consider more examples of non-reductive self-dual algebras and study the $\sigma$-model string backgrounds that correspond to them.

Any non-reductive (indecomposable) self-dual algebra can be constructed, starting from an Abelian algebra, by a sequence of construction steps, each of which is either an (orthogonal) direct product or a procedure called "double extension" [17]. If such an algebra can be obtained through a Wigner contraction, it is necessarily a double extension of an Abelian algebra, i.e., it is a result of a single-step sequence. All the algebras used so far to construct WZNW models are double extensions of Abelian algebras and, therefore, possibly can be obtained through a Wigner contraction (for some of them this was explicitly shown [5] 15]). One might have suspected that all non-reductive, self-dual algebras can be obtained through a Wigner contraction. If this was true, it would have a significant implication on the structure of such algebras and the WZNW models based on them. It turns out, however, that this is not true.

In fact, we present an infinite family of indecomposable, non-reductive, self-dual algebras $\left\{\mathcal{A}_{3 m}\right\}$ [18], that (except $\mathcal{A}_{3}$ and $\mathcal{A}_{6}$ ) are not double extensions of Abelian algebras and, therefore, cannot be obtained through a Wigner contraction $\left(\mathcal{A}_{6}\right.$ is also unobtainable through a Wigner contraction). The algebra

$$
\mathcal{A}_{n} \equiv \operatorname{sp}\left\{T_{i}\right\}_{0 \leq i \leq n}
$$

is defined by the Lie bracket

$$
\left[T_{i}, T_{j}\right]=\left\{\begin{array}{cc}
(\widehat{i-j}) T_{i+j} & i+j \leq n \\
0 & \text { otherwise }
\end{array}\right.
$$


where $\hat{i} \equiv i \bmod 3$ is chosen to be in $\{-1,0,1\}$. When $\hat{n}=0$, the metric

$$
\left(T_{i}, T_{j}\right)=\delta_{i+j-n}+b \delta_{i} \delta_{j}
$$

is an invariant metric on $\mathcal{A}_{n}$ (for arbitrary $b$ ). We construct WZNW and gauged WZNW models based on the first two algebras in this series: $\mathcal{A}_{3}$ and $\mathcal{A}_{6}$.

The paper is organized as follows: in section 2, we describe the family $\left\{\mathcal{A}_{n}\right\}$ of selfdual algebras. While constructing the gauged WZNW examples, we encountered some phenomena which are very common when the algebras are not semi-simple and seldom (or never) appear otherwise. In section 3, we analyze some of them in a general setting: the appearance of constraints; singular gauging (when the restriction of the metric to the gauged subalgebra is singular() and the gauging of a central subgroup. In section 4, we construct WZNW and gauged WZNW models based on $\mathcal{A}_{3}$ and $\mathcal{A}_{6}$. In Appendix A, we collect some parenthetical remarks complementing the main text and, in Appendix $\mathrm{B}$, we list the geometrical information of all the $\sigma$ models derived in section 4 . For all these models the one-loop beta functions [20] vanish and the central charge is equal to the dimension of the $\sigma$-model target manifold.

\section{A New Family of Solvable Self-Dual Lie Algebras}

In this section we describe the family $\left\{\mathcal{A}_{n}\right\}$ of self-dual algebras, as obtained in [18]. The main results are described in the introduction and the reader interested in the physical results may skip directly to section 3. We start, in subsection 2.1 with a review of the two methods for constructing self-dual Lie algebras - a double extension and a Wigner contraction. In subsection 2.2 we define the algebras $\mathcal{A}_{n}$ and prove that (for $\hat{n}=0$ ) these are indeed self-dual Lie algebras. In subsection 2.3 we find all the ideals of $\mathcal{A}_{n}$. This result is used in the last subsection, where we check which of these algebras is a double extension of an Abelian algebra or a result of a Wigner contraction.

\subsection{The Construction of Self-Dual Lie algebras}

A self-dual Lie algebra $\mathcal{A}$ is a Lie algebra that admits an invariant metric, i.e. a symmetric non-degenerate bilinear form $(\cdot, \cdot)$ which is invariant under the adjoint action of the corresponding group:

$$
\left(g x_{1} g^{-1}, g x_{2} g^{-1}\right)=\left(x_{1}, x_{2}\right), \quad \forall x_{i} \in \mathcal{A}
$$

for any $g$ in the group, or equivalently:

$$
\left(\left[y, x_{1}\right], x_{2}\right)=-\left(x_{1},\left[y, x_{2}\right]\right), \quad \forall x_{i} \in \mathcal{A}
$$

\footnotetext{
${ }^{4}$ For examples of the special case of null gauging, when the metric on the gauged subalgebra vanishes, see [19] and references therein.
} 
for any $y \in \mathcal{A}$. The best known families of self-dual algebras are the semi-simple algebras (where the (unique) invariant metric is the Killing form) and the Abelian algebras (for which every metric is trivially invariant). However, these are not the only ones. In this section we are concerned with the search for self dual algebras that are neither semisimple nor Abelian. Given two self dual algebras, their direct sum equipped with the natural direct sum metric, is also self dual (this construction will be called an orthogonal direct sum), therefore, in the construction of self dual algebras, the non-trivial task is to find the indecomposable ones, i.e. algebras that are not orthogonal direct sums. It has been shown [17] that any indecomposable self-dual Lie algebra, which is neither simple nor one dimensional, is a double extension of a smaller self-dual Lie algebra (see also [15]), therefore, one may attempt to use the procedure of double extension for actual construction of new indecomposable self-dual Lie algebras.

The double extension of a self-dual Lie algebra $\mathcal{A}$ by another Lie algebra $\mathcal{B}$ (not necessarily self-dual) can be seen as a two-step process. The first step is to combine them to a semi-direct sum

$$
\mathcal{S}=\mathcal{B} \in \mathcal{A}
$$

in such a way that the metric in $\mathcal{A}$ will be invariant also under the action of $\mathcal{B}$. For this, one needs, in addition to the algebras $\mathcal{A}$ and $\mathcal{B}$, an action (representation) of $\mathcal{B}$ in $\mathcal{A}$

$$
y: x \rightarrow[y, x], \quad\left[\left[y_{1}, y_{2}\right], x\right]=\left[y_{1},\left[y_{2}, x\right]\right]-\left[y_{2},\left[y_{1}, x\right]\right]
$$

that will satisfy the mixed Jacobi identities

$$
\left[y,\left[x_{1}, x_{2}\right]\right]=\left[\left[y, x_{1}\right], x_{2}\right]+\left[x_{1},\left[y, x_{2}\right]\right]
$$

and the invariance condition (2.2) (here $\left.x, x_{i} \in \mathcal{A}, y, y_{a} \in \mathcal{B}\right)$. Given bases $\left\{x_{i}\right\}$ and $\left\{y_{a}\right\}$ for $\mathcal{A}$ and $\mathcal{B}$ respectively, the Lie bracket of $\mathcal{S}$ is of the form

\begin{tabular}{c|cc}
{$[\cdot, \cdot]$} & $y_{b}$ & $x_{j}$ \\
\hline$y_{a}$ & $f_{a b}{ }^{c} y_{c}$ & $f_{a j}{ }^{k} x_{k}$ \\
$x_{i}$ & $-f_{b i}{ }^{k} x_{k}$ & $f_{i j}{ }^{k} x_{k}$
\end{tabular}

$f_{i j}{ }^{k}$ and $f_{a b}{ }^{c}$ are the structure constants of $\mathcal{A}$ and $\mathcal{B}$ respectively and as such satisfy the Jacobi Identities; $f_{i j}{ }^{k}$ satisfies an additional identity

$$
f_{i j}{ }^{l} \Omega_{l k}+f_{i k}^{l} \Omega_{l j}=0
$$

expressing the invariance (2.2) of the metric $\Omega_{i j}=\left(x_{i}, x_{j}\right)$ on $\mathcal{A} ; f_{a j}{ }^{k}$ represent the action of $y_{a}$ in $\mathcal{A}$ and identities (2.4), (2.5) and (2.2) take respectively the form

$$
f_{a b}^{c} f_{c k}^{l}=f_{a m}^{l} f_{b k}^{m}-f_{b m}^{l} f_{a k}^{m}
$$

\footnotetext{
${ }^{5}$ By this we mean that the vector space $\mathcal{S}$ is a direct sum of the vector spaces $\mathcal{B}$ and $\mathcal{A}, \mathcal{B}$ is a subalgebra of $\mathcal{S}:[\mathcal{B}, \mathcal{B}] \subset \mathcal{B}$ and $\mathcal{A}$ is an ideal of $\mathcal{S}:[\mathcal{S}, \mathcal{A}] \subset \mathcal{A}$.
} 


$$
f_{a k}^{l} f_{i j}{ }^{k}=f_{a i}{ }^{k} f_{k j}^{l}+f_{i k}^{l} f_{a j}{ }^{k}
$$

and

$$
f_{a j}{ }^{l} \Omega_{l k}+f_{a k}{ }^{l} \Omega_{l j}=0 .
$$

The second step is the extension of $\mathcal{S}$ by an Abelian algebra $\mathcal{B}^{*}$ with $\operatorname{dim} \mathcal{B}^{*}=\operatorname{dim} \mathcal{B}$. This step is completely determined by the first one (the Lie bracket in $\mathcal{S}$ and the metric on $\mathcal{A})$ and in an appropriate basis $\left\{z^{a}\right\}$ for $\mathcal{B}^{*}$ the Lie bracket of the resulting algebra, which will be denoted by $\mathcal{D}$, is

\begin{tabular}{c|ccc}
{$[\cdot, \cdot]$} & $y_{b}$ & $x_{j}$ & $z^{b}$ \\
\hline$y_{a}$ & $f_{a b}{ }^{c} y_{c}$ & $f_{a j}{ }^{k} x_{k}$ & $-f_{a c}{ }^{b} z^{c}$ \\
$x_{i}$ & $-f_{b i}{ }^{k} x_{k}$ & $f_{i j}{ }^{k} x_{k}+f_{c i}{ }^{k} \Omega_{k j} z^{c}$ & 0 \\
$z^{a}$ & $f_{b c}{ }^{a} z^{c}$ & 0 & 0
\end{tabular}

This algebra has an invariant metric, which in the above basis is

$$
(\cdot, \cdot)=\left(\begin{array}{ccc}
\omega_{a b} & 0 & \delta_{a}^{b} \\
0 & \Omega_{i j} & 0 \\
\delta_{b}^{a} & 0 & 0
\end{array}\right),
$$

where $\omega_{a b}$ is some invariant symmetric bilinear form on $\mathcal{B}$ (possibly degenerate; e.g. the Killing form or zero).

The theorem proved in [17] states that an indecomposable self-dual Lie algebra, which is neither simple nor one dimensional, is a double extension of a self-dual algebra (with smaller dimension) by a simple or one-dimensional algebra. Although this is a very important and useful result for a general study of these algebras, its straightforward application to an actual construction is cumbersome, as we now explain. To doubleextend a self-dual Lie algebra $\mathcal{A}$, one needs (a Lie algebra $\mathcal{B}$ of) linear transformations in $\mathcal{A}$ satisfying (2.5) and (2.2). One could, of course, take the trivial action: $y: x \rightarrow 0$, but the resulting algebra $\mathcal{D}$ is decomposable - the original algebra $\mathcal{A}$ factorizes out:

$$
\mathcal{D}=\mathcal{A} \stackrel{\perp}{\oplus}\left(\mathcal{B} \in \mathcal{B}^{*}\right) .
$$

Moreover, it was shown in 15$]$ that even if $\mathcal{B}$ acts non-trivially but its action is through inner derivations (i.e. the action of each $y \in \mathcal{B}$ coincides with the adjoint action of an element $\hat{y} \in \mathcal{A}: y: x \rightarrow[\hat{y}, x])$, the result is also decomposable. This means that for the construction of an indecomposable double extension, one needs knowledge about the outer (non-inner) derivations in $\mathcal{A}$ and such information is not available in general. In the absence of general results, the suitable transformations must be found by direct calculation. Given $\mathcal{A}$ and $(\cdot, \cdot)_{\mathcal{A}}$, one must find solutions $f_{a j}{ }^{k}$ of the (linear) equations (2.9) and (2.10) and identify among them, by elimination, those that correspond to inner derivations. 
Another method for constructing new self-dual Lie algebras is by performing a Wigner contraction [16] (this was proposed, in the context of WZNW models, in [5). The initial data for this construction consists of a self dual Lie algebra $\mathcal{S}_{0}$ and a sub algebra $\mathcal{B}_{0}$ of $\mathcal{S}_{0}$ such that the restriction of the metric $(\cdot, \cdot)$ on $\mathcal{S}_{0}$ to $\mathcal{B}_{0}$ is non-degenerate. The last condition is equivalent to

$$
\mathcal{S}_{0}=\mathcal{B}_{0} \oplus \mathcal{B}_{0}^{\perp}
$$

$\left(\mathcal{B}_{0}^{\perp}\right.$ is the orthogonal complement of $\mathcal{B}_{0}$ with respect to the metric), therefore, bases $\left\{b_{a}^{0}\right\}$ for $\mathcal{B}_{0}$ and $\left\{a_{i}\right\}$ for $\mathcal{B}_{0}^{\perp}$ combine to a basis for $\mathcal{S}_{0}$. In this basis, the Lie bracket in $\mathcal{S}_{0}$ has the general form

$$
\begin{gathered}
{\left[a_{i}, a_{j}\right]=f_{i j}{ }^{k} a_{k}+f_{i j}{ }^{c} b_{c}^{0},} \\
{\left[b_{a}^{0}, a_{j}\right]=f_{a j}{ }^{k} a_{k} \quad, \quad\left[b_{a}^{0}, b_{b}^{0}\right]=f_{a b}{ }^{c} b_{c}^{0}}
\end{gathered}
$$

and the metric is

$$
(\cdot, \cdot)=\left(\begin{array}{cc}
\Omega_{i j} & 0 \\
0 & \Omega_{a b}
\end{array}\right) .
$$

One now performs a Wigner contraction [16] of $\mathcal{S}_{0} \oplus \mathcal{B}_{1}$ (where $\mathcal{B}_{1}$ is isomorphic to $\mathcal{B}_{0}$ and commutes with $\mathcal{S}_{0}$ ): define

$$
x_{i} \equiv \epsilon a_{i}, \quad y_{a} \equiv b_{a}^{0}+b_{a}^{1}, \quad z_{a} \equiv \frac{1}{2} \epsilon^{2}\left(b_{a}^{0}-b_{a}^{1}\right),
$$

express the Lie brackets in terms of the new variables and take the limit $\epsilon \rightarrow 0$. Since this is a singular limit, one obtains a new algebra $\mathcal{D}$ (not isomorphic to $\mathcal{S}_{0} \oplus \mathcal{B}_{1}$ ) with the following Lie brackets:

\begin{tabular}{c|ccc}
{$[\cdot, \cdot]$} & $y_{b}$ & $x_{j}$ & $z_{b}$ \\
\hline$y_{a}$ & $f_{a b}{ }^{c} y_{c}$ & $f_{a j}{ }^{k} x_{k}$ & $f_{a b}{ }^{c} z_{c}$ \\
$x_{i}$ & $-f_{b i}{ }^{k} x_{k}$ & $f_{i j}{ }^{c} z_{c}$ & 0 \\
$z_{a}$ & $-f_{b a}{ }^{c} z_{c}$ & 0 & 0
\end{tabular}

To obtain an invariant metric for $\mathcal{D}$ one starts with the natural invariant metric on $\mathcal{S}_{0} \oplus \mathcal{B}_{1}$, that in the basis $\left\{b_{a}^{0}, a_{i}, b_{a}^{1}\right\}$ is

$$
(\cdot, \cdot)_{\epsilon}=\left(\begin{array}{ccc}
\beta_{0} \Omega_{a b} & 0 & 0 \\
0 & \beta_{0} \Omega_{i j} & 0 \\
0 & 0 & \beta_{1} \Omega_{a b}
\end{array}\right)
$$

\footnotetext{
${ }^{6}$ The structure constants satisfy the Jacobi identities and the identities expressing the invariance of the metric. In particular, the vanishing of $f_{a j}{ }^{c}$ follows from the existence of a non-singular invariant metric:

$$
\left(b_{c}^{0},\left[b_{a}^{0}, a_{j}\right]\right)=\left(\left[b_{c}^{0}, b_{a}^{0}\right], a_{j}\right)=0 .
$$
}


(with arbitrary $\beta_{0}, \beta_{1}$ ). In the limit $\epsilon \rightarrow 0$ one obtains:

$$
(\cdot, \cdot)_{0}=\left(\begin{array}{ccc}
\beta^{\prime} \Omega_{a b} & 0 & \beta \Omega_{a b} \\
0 & \beta \Omega_{i j} & 0 \\
\beta \Omega_{a b} & 0 & 0
\end{array}\right)
$$

(in the basis $\left.\left\{y_{a}, a_{i}, z_{a}\right\}\right)$ where]

$$
\beta=\lim _{\epsilon \rightarrow 0} \frac{1}{2} \epsilon^{2}\left(\beta_{0}-\beta_{1}\right) \quad, \quad \beta^{\prime}=\lim _{\epsilon \rightarrow 0}\left(\beta_{0}+\beta_{1}\right)
$$

and this form is invariant (by continuity) and, for $\beta \neq 0$, non-degenerate.

The resemblance of the resulting algebra to the one obtained by double extension is apparent and indeed, using the metric to raise and lower indexes, one immediately identifies it as the double extension of an Abelian algebra $\mathcal{A}=\operatorname{sp}\left\{x_{i}\right\}$ by $\mathcal{B}=\operatorname{sp}\left\{y_{a}\right\}$ [15] (where $\operatorname{sp}\left\{x_{i}\right\}$ denotes the linear span of the set $\left\{x_{i}\right\}$ ). However, this method has a clear advantage. Unlike double extension, the initial data needed is very simple and generally available, therefore, the method can be easily used to find many new non-trivial self-dual algebras.

A natural question is if there are non-semi-simple, indecomposable, self-dual algebras, that cannot be obtained by a Wigner contraction. Any self-dual Lie algebra obtained through a Wigner contraction can be obtained from an Abelian algebra by a single doubleextension, therefore, this question is closely related to the problem of finding (non-semisimple, indecomposable, self-dual) algebras that their construction out of simple and one dimensional algebras involves more than one double extension [ and in this sense, are called deeper algebras [15]. As explained above, deeper algebras are not easy to find. In fact, among the self dual algebras with dimension at most 5 (enumerated in [12]), none is deeper than a double extension of an Abelian algebra. In the rest of this section we introduce and explore a family of deeper algebras.

\subsection{The Algebra $\mathcal{A}_{n}$}

Consider a vector space, equipped with the basis $\left\{T_{i}\right\}_{i \in \mathbb{Z}}$ and the following "Lie bracket" 18]:

$$
\left[T_{i}, T_{j}\right]=(\widehat{i-j}) T_{i+j}
$$

where $\hat{i} \equiv i \bmod 3$ is chosen to be in $\{-1,0,1\}$. The map $i \rightarrow \hat{i}$ is almost a ring homomorphism $\mathbb{Z} \rightarrow \mathbb{Z}$ : it preserves multiplication

$$
\widehat{(i j)}=\hat{i} \hat{j}
$$

\footnotetext{
${ }^{7}$ Note that to obtain a non-degenerate metric, $\beta_{0}$ and $\beta_{1}$ must depend non-trivially on $\epsilon$. In fact they must diverge in the limit $\epsilon \rightarrow 0$.

${ }^{8}$ One might also consider a double extension of a reductive algebra $\mathcal{A}$ i.e. an orthogonal direct sum of Abelian and semi-simple algebras. However, as shown in [15], the semi-simple factor of $\mathcal{A}$ factorizes also in the result $\mathcal{D}$ (because a semi-simple algebra does not have outer derivations), therefore, the result in this case is decomposable.
} 
and almost preserves addition?:

$$
\begin{gathered}
(\widehat{i+j})=(\hat{i+\hat{j}}) \quad, \quad \widehat{(-i)}=-\hat{i} \\
(\hat{i-j})=0 \Longleftrightarrow \hat{i}=\hat{j}
\end{gathered}
$$

(but note that $\hat{1}+\hat{1} \neq(\widehat{1+1}))$. These are the properties that will be used in the following. Particularly useful will be the property

$$
\hat{i}=\hat{j} \Longleftrightarrow(\hat{i+k})=(\widehat{j+k})
$$

which follows from (2.26).

The bracket is manifestly anti-symmetric so to obtain a Lie algebra, there remains to verify the Jacobi identity. Since

$$
\left[\left[T_{i}, T_{j}\right], T_{k}\right]=\hat{c}_{i j k} T_{i+j+k} \quad, \quad c_{i j k} \equiv(i-j)(i+j-k),
$$

the Jacobi identity takes the form

$$
\hat{c}_{i j k}+\hat{c}_{j k i}+\hat{c}_{k i j}=0
$$

This identity holds without the 'hats', therefore, by (2.25),

$$
\hat{c}_{i j k}+\hat{c}_{j k i}+\hat{c}_{k i j}=0 \bmod 3,
$$

so 2.29$)$ can be false only when

$$
\hat{c}_{i j k}=\hat{c}_{j k i}=\hat{c}_{k i j}= \pm 1 \text {. }
$$

$\hat{c}_{i j k}=1$ is equivalent to $(\widehat{i-j})=(i+\widehat{j}-k)= \pm 1$ and, therefore, also to

$$
i=j \pm 1 \bmod 3 \quad, \quad k=-j \bmod 3
$$

and this cannot hold simultaneously for all the tree cyclic permutations of $\{i j k\}$. Replacing $i \leftrightarrow j$ one obtains the same result for $\hat{c}_{i j k}=-1$. Therefore, the Jacobi identity holds and the above algebra is indeed a Lie algebra (over the integers)

Let us consider the subalgebra

$$
\mathcal{A}_{\infty} \equiv \operatorname{sp}\left\{T_{i}\right\}_{i \geq 0}
$$

\footnotetext{
${ }^{9}$ The $\Longleftarrow$ direction of $(2.26$ follows from $(2.25$, but for the other direction 2.25 ) only implies

$$
(\widehat{i-j})=0 \Longrightarrow \hat{i}-\hat{j}=0 \bmod 3
$$

and the stronger result $\hat{i}-\hat{j}=0$ follows from the fact that $|\hat{i}-\hat{j}|$ is always at most 2 . When $(\widehat{i-j}) \neq 0$ this reasoning breaks down and indeed we have e.g. $\hat{2}-\hat{1} \neq(\widehat{2-1})$.

${ }^{10}$ In Appendix A.1 we comment about possible generalizations of this algebra.
} 
Dividing by the ideal $\operatorname{sp}\left\{T_{i}\right\}_{i>n}$ (for some positive integer $n$ ), one obtains the finite dimensional Lie algebra

$$
\mathcal{A}_{n} \equiv \operatorname{sp}\left\{T_{i}\right\}_{0 \leq i \leq n}
$$

with the Lie bracket

$$
\left[T_{i}, T_{j}\right]=\left\{\begin{array}{cc}
(\widehat{i-j}) T_{i+j} & i+j \leq n \\
0 & \text { otherwise }
\end{array}\right.
$$

From now on we restrict our attention to such an algebra. It is a solvable ${ }^{\text {II }}$ algebra, $T_{0}$ being the only non-nilpotent generator and it possesses a $\mathbb{Z}$-grading: $\operatorname{deg}\left(T_{i}\right)=i$ (inherited from the original infinite-dimensional algebra.)

We would like to find an invariant metric $(\cdot, \cdot)$ on $\mathcal{A}_{n}$. Using (1.1), the invariance condition

$$
\left(\left[T_{k}, T_{i}\right], T_{j}\right)+\left(T_{i},\left[T_{k}, T_{j}\right]\right)=0
$$

takes the form

$$
(\widehat{k-i})\left(T_{k+i}, T_{j}\right)+(\widehat{k-} j)\left(T_{k+j}, T_{i}\right)=0
$$

(here $T_{i} \equiv 0$ for $i>0$ ) and, in particular, for $k=0$ :

$$
(\widehat{(-i)} \widehat{+}(\widehat{-j}))\left(T_{i}, T_{j}\right)=0
$$

which, by eqs. (2.25,2.26), is equivalent to

$$
(\widehat{i+j})\left(T_{i}, T_{j}\right)=0
$$

This means that two out of each three "reversed" (right-up-to-left-down) diagonals vanish. Let us look for a metric with only one non-vanishing diagonal. To obtain a non-degenerate form, this must be the central diagonal and according to (2.35), this is possible only for $\hat{n}=0$. We, therefore, concentrate on this case and consider a metric of the form

$$
\left(T_{i}, T_{j}\right)=\omega_{j} \delta_{i+j, n} \quad, \quad \omega_{n-j}=\omega_{j} \neq 0
$$

For such a metric the invariance condition (2.33) takes the form

$$
(\widehat{k-i}) \omega_{j}+(\widehat{k-j}) \omega_{i}=0, \quad \forall i+j+k=n
$$

and using $\hat{n}=0$, one obtains

$$
(2 \widehat{i+j}) \omega_{j}+(2 \widehat{j+i}) \omega_{i}=0
$$

First we take $\hat{j}=0$ which gives

$$
\hat{i}\left(\omega_{i}+\hat{2} \omega_{j}\right)=0 \text {. }
$$

\footnotetext{
${ }^{11}$ Solvability of a Lie algebra $\mathcal{A}$ is defined as follows: One defines recursively $\mathcal{A}^{k+1}=\left[\mathcal{A}^{k}, \mathcal{A}^{k}\right] ; \mathcal{A}$ is solvable iff for some $k, \mathcal{A}^{k}=0$.
} 
and this implies (since $\hat{2} \neq 0$ )

$$
\omega_{i}=\left\{\begin{array}{cc}
\omega_{i}=-\hat{2} \omega_{0} & \hat{i} \neq 0 \\
\omega_{i}=\omega_{0} & \hat{i}=0 .
\end{array}\right.
$$

Using this result we take $\hat{i}, \hat{j} \neq 0$ in (2.38) and obtain

$$
\hat{2} \cdot \hat{3}(\hat{i+j}) \omega_{0}=0
$$

which is satisfied, since ${ }^{\mathbb{3}}=0 .-\hat{2}=1$, therefore, we have $\omega_{i}=\omega_{0}, \forall i$. To summarize, we proved:

\section{Lemma:}

$A$ (non-degenerate) invariant metric on $\mathcal{A}_{n}$ with only one (reversed) diagonal exists iff $\hat{n}=0$ and it is proportional to

$$
\left(T_{i}, T_{j}\right)=\delta_{i+j-n}
$$

Note that one can add to the metric a multiple of the Killing form, obtaining

$$
\left(T_{i}, T_{j}\right)=\delta_{i+j-n}+b \delta_{i} \delta_{j}
$$

(with $b$ arbitrary). The appearance of the second term can also be seen as a result of the (automorphic) change of basis

$$
T_{0} \rightarrow T_{0}+\frac{1}{2} b T_{n}
$$

\subsection{The Ideals in $\mathcal{A}_{n}$}

In this subsection we continue to analyze the algebra $\mathcal{A}_{n}$, looking for all its ideals and concluding that the only ideals are of the form

$$
\mathcal{A}_{m, n} \equiv \operatorname{sp}\left\{T_{i}\right\}_{i=m}^{n} .
$$

This will be important in the next subsection, where we will check if these algebras are double extensions of Abelian algebras. The grading on $\mathcal{A}_{n}\left(\operatorname{deg}\left(T_{i}\right)=i\right)$ will play a central role in the following and will be called "charge". The adjoint action of $T_{i}$ increases the charge by $i$. Note that there are only positive charges, so that the adjoint action cannot decrease the charge. This proves that $\mathcal{A}_{m, n}$ (for any $m$ ) is indeed an ideal.

Let $\mathcal{J}$ be an ideal in $\mathcal{A}_{n}$. We choose a basis for $\mathcal{J}$ such that each element has a different minimal charge (this can be easily accomplished) and, therefore, can be labeled by it. We, therefore, have (after an appropriate normalization):

$$
\mathcal{J}=\operatorname{sp}\left\{S_{\alpha}\right\}, \quad S_{\alpha}-T_{\alpha} \in \mathcal{A}_{\alpha+1, n} .
$$

\footnotetext{
${ }^{12}$ This is where the derivation stops being valid for the Virasoro algebra (mentioned in Appendix A.1, where possible generalizations of the algebra (2.23) are discussed).
} 
Isolating in $\mathcal{J}$ the maximal ideal of the form $\mathcal{A}_{m, n}$, we obtain:

$$
\mathcal{J}=\operatorname{sp}\left\{S_{\alpha}\right\}_{\alpha \in \mathbf{A}} \bigoplus \mathcal{A}_{m, n} \quad, \quad m-1 \notin \mathbf{A}
$$

Observe that this implies that for any element in $\mathcal{J}$ that is not in $\mathcal{A}_{m, n}$, its minimal charge is in $\mathbf{A}$.

The choice $\mathbf{A}=\emptyset$ (the empty set) corresponds to the "trivial" solution $\mathcal{J}=\mathcal{A}_{m, n}$. In the following we look for other solutions, i.e. with $\mathbf{A} \neq \emptyset$. This also implies $\max (\mathbf{A})<$ $m-1$. We are going to explore the restrictions on the $S_{\alpha}$ 's implied by the claim that $\mathcal{J}$ is an ideal in $\mathcal{A}_{n}$. Since $\mathcal{A}_{m, n}$ is an ideal by itself, the only restrictions come from

$$
\left[T_{i}, S_{\alpha}\right] \in \mathcal{J} \quad \forall \alpha \in \mathbf{A} \quad, \quad i=0, \ldots, n
$$

$\mathcal{J}$ contains all terms with charge at least $m$, therefore, restrictions will arise only in terms in the commutator with smaller charge. For $i \geq m-\alpha$ there are no such terms. As the charge $i$ decreases, there will be more non-trivial terms, therefore, we will start from the higher charges.

For $i=m-\alpha-1$ we have (in the following, " $\simeq$ " means "equality up to an element of $\left.\mathcal{A}_{m, n} "\right)$ :

$$
\left[T_{m-\alpha-1}, S_{\alpha}\right] \simeq\left[T_{m-\alpha-1}, T_{\alpha}\right]=(m-\widehat{2 \alpha}-1) T_{m-1}
$$

(here and in other similar cases the hat should be applied to the whole expression between parenthesis). $T_{m-1} \notin \mathcal{J}$ (otherwise $\mathcal{A}_{m-1, n} \subset \mathcal{J}$ ), therefore,

$$
(m-\widehat{2 \alpha}-1)=0 \text {. }
$$

Using eqs. (2.25,2.26), this is equivalent to

$$
\hat{\alpha}=-\widehat{(2 \alpha)}=-(\widehat{m-1})
$$

and since this is true for all $\alpha \in \mathbf{A}$, we also have

$$
\hat{\alpha}_{1}=\hat{\alpha}_{2} \quad \forall \alpha_{1}, \alpha_{2} \in \mathbf{A}
$$

Next, for $i=m-\alpha-2$ we have (using eqs. (2.49) and (2.25))

$$
\left[T_{m-\alpha-2}, S_{\alpha}\right] \simeq\left[T_{m-\alpha-2}, T_{\alpha}+s_{\alpha}^{\alpha+1} T_{\alpha+1}\right]=-T_{m-2}+s_{\alpha}^{\alpha+1} T_{m-1}
$$

This implies that $m-2$ is a minimal charge of an element of $\mathcal{J}$, therefore, $m-2 \in \mathbf{A}$. Substituting $\alpha=m-2$ in (2.51) we obtain

$$
\left[T_{0}, S_{m-2}\right] \simeq-T_{m-2}+s_{m-2}^{m-1} T_{m-1} \simeq-S_{m-2}+2 s_{m-2}^{m-1} T_{m-1}
$$

and this implies $s_{m-2}^{m-1}=0$, so with no loss of generality, we can choose

$$
S_{m-2}=T_{m-2}
$$


Finally, for $i=m-\alpha-3$ and $m-2>\alpha \in \mathbf{A}$ we have

$$
\left[T_{m-\alpha-3}, S_{\alpha}\right] \simeq\left[T_{m-\alpha-3}, T_{\alpha}+s_{\alpha}^{\alpha+1} T_{\alpha+1}+s_{\alpha}^{\alpha+2} T_{\alpha+2}\right]=T_{m-3}+s_{\alpha}^{\alpha+2} T_{m-1}
$$

and as before this should imply that $m-3 \in \mathbf{A}$ (being the minimal charge of an element of $\mathcal{J}$ ). However, according to eq. (2.50), this is impossible since $m-2 \in \mathbf{A}$. Therefore, A contains no elements other then $m-2$ and $\mathcal{J}$ is of the form

$$
\mathcal{J}=\operatorname{sp}\left\{T_{m-2}\right\} \oplus \mathcal{A}_{m, n}
$$

A straightforward check (or use of eq. (2.49)) shows that this is indeed an ideal iff $\hat{m}=0$. Is this ideal really non-trivial? It turns out that it is not! To see this, consider the (nonsingular) linear map defined by $T_{i} \mapsto T_{i}^{\prime} \equiv-T_{i+\hat{i}}$. Since $\hat{m}=0$, this map transforms $\mathcal{J}$ to $\mathcal{A}_{m-1, n}$.

$$
\left[T_{i}^{\prime}, T_{j}^{\prime}\right]=-(\widehat{i-j}) T_{i+j+(\hat{i}+\hat{j})}=-(\widehat{i-j}) T_{i+j+(\widehat{i+j})}=(\widehat{i-j}) T_{i+j}^{\prime}
$$

(the second equality follows from the fact that for $(\widehat{i-j}) \neq 0,(\hat{i+j})=\hat{i}+\hat{j})$, therefore, this map is an automorphism of Lie algebras, which means that $\mathcal{J}=\operatorname{sp}\left\{T_{m-2}\right\} \oplus \mathcal{A}_{m, n}$ is automorphic to $\mathcal{A}_{m-1, n}$.

\section{$2.4 \mathcal{A}_{n}$ as a Deeper algebra}

Now we are ready to check how the self-dual algebras found above fit into the general picture described in the beginning of this section. We consider here the case $\hat{n}=0$. The list of the ideals found in the previous subsection implies that none of these algebras is decomposable (i.e. expressible as an orthogonal direct sum) []. Among the indecomposable self-dual algebras, we have the following inclusion relations:

\section{\{ Indecomposable, Self-Dual Algebras \} \\ $\cup$ \\ \{ (Single) Double-Extensions of Abelian Algebras \} \\ $\cup$ \\ \{ Algebras obtainable by a Wigner contraction \}}

We will show that these are strict inclusions, i.e., all the three sets are distinct. Explicitly we will show here that among the algebras $\mathcal{A}_{n}, \mathcal{A}_{3}$ can be obtained by a Wigner contraction, $\mathcal{A}_{6}$ is a double extension of an Abelian algebra but cannot be obtained by a Wigner contraction, and the rest are deeper algebras i.e. they are not double extensions

\footnotetext{
${ }^{13}$ This means that they should be expressible as double extensions by the one dimensional algebra, and this structure can be indeed easily identified:

$$
\mathcal{B}=\operatorname{sp}\left\{T_{0}\right\} \quad, \quad \mathcal{B}^{*}=\operatorname{sp}\left\{T_{n}\right\} \quad, \quad \mathcal{A}=\mathcal{A}_{1, n} / \mathcal{B}^{*}
$$


of Abelian algebras and, therefore, in particular, they cannot be obtained by a Wigner contraction.

We start by trying to identify in $\mathcal{A}_{n}$ the structure of a double extension of an Abelian algebra. The Lie product in an algebra $\mathcal{D}$, with such a structure (table (2.11)) is of the following form

\begin{tabular}{c|ccc}
{$[\cdot, \cdot]$} & $\mathcal{B}$ & $\mathcal{A}$ & $\mathcal{B}^{*}$ \\
\hline $\mathcal{B}$ & $\mathcal{B}$ & $\mathcal{A}$ & $\mathcal{B}^{*}$ \\
$\mathcal{A}$ & $\mathcal{A}$ & $\mathcal{B}^{*}$ & 0 \\
$\mathcal{B}^{*}$ & $\mathcal{B}^{*}$ & 0 & 0
\end{tabular}

where $\mathcal{A}=\operatorname{sp}\left\{x_{i}\right\}, \mathcal{B}=\operatorname{sp}\left\{y_{a}\right\}$ and $\mathcal{B}^{*}=\operatorname{sp}\left\{z_{a}\right\}$. In this table we recognize two properties of $\mathcal{D}$

1. $\mathcal{D}$ is a semi direct sum of $\mathcal{B}$ and the ideal $\mathcal{J}=\mathcal{A}+\mathcal{B}^{*}: \mathcal{D}=\mathcal{B} \in \mathcal{J}$;

2. $[\mathcal{J}, \mathcal{J}] \subset \mathcal{B}^{*}$, therefore, $\operatorname{dim}[\mathcal{J}, \mathcal{J}] \leq \operatorname{dim} \mathcal{B}^{*}=\operatorname{dim} \mathcal{B}$.

Consider the first property. The candidates for the ideal $\mathcal{J}$ were found in the previous subsection. It was shown that $\mathcal{J}=\mathcal{A}_{m, n}$ (possibly after an automorphic change of basis $\left\{T_{i}\right\}$ ). Following the same approach, we choose a basis $\left\{R_{i}\right\}_{i=0}^{m-1}$ for $\mathcal{B}$ such that $i$ is the minimal charge of $R_{i}$. $\left[T_{m-1}, T_{m-2}\right]=T_{2 m-3}$ and $2 m-3<n\left(\right.$ since $\left.\operatorname{dim} \mathcal{A}_{n} \geq 2 \operatorname{dim} \mathcal{B}\right)$, therefore, $\left[R_{m-1}, R_{m-2}\right] \neq 0$ and its minimal charge is $2 m-3$. $\mathcal{B}$ is closed under the Lie bracket and $\mathcal{B} \cap \mathcal{J}=\{0\}$, therefore, $\left[R_{m-1}, R_{m-2}\right] \notin \mathcal{J}$, which implies that $2 m-3<m$. This leaves us with $m=1$ or 2 .

As for the second property, we have

$$
\operatorname{dim}[\mathcal{J}, \mathcal{J}] \leq \operatorname{dim} \mathcal{B}=m
$$

One can easily verify that

$$
\left[\mathcal{A}_{m, n}, \mathcal{A}_{m, n}\right]=\mathcal{A}_{2 m+1, n}
$$

therefore, eq. (2.59) implies $n \leq 3 m$. On the other hand $n+1 \geq 2 m$ (since $\operatorname{dim} \mathcal{A}_{n} \geq$ $2 \operatorname{dim} \mathcal{B})$. Recalling that $\hat{n}=0$, We obtain three possibilities:

$$
(m, n)=(1,3),(2,3),(2,6)
$$

and a direct check confirms that each of them indeed corresponds to a double extension of an Abelian algebra (in the second possibility this is the zero-dimensional algebra). Observe that there are more than one way to represent an algebra as a double extension. Moreover, $\mathcal{A}_{6}$ can be obtained both by extending an Abelian algebra (with $m=2$ ) and

\footnotetext{
${ }^{14}$ The value $m=0$ is also a possibility but it is not interesting. It corresponds to $\operatorname{dim} \mathcal{B}=0$. As a double extension it means not to do anything - remaining with the (Abelian) algebra $\mathcal{A}$ one started with. As a Wigner contraction it means that, starting with some self-dual Lie algebra $\mathcal{S}_{0}$, all we did is to set its Lie bracket to 0 , so that we end up with the Abelian Lie algebra of the same dimension, which is trivially self dual. In the present context, this corresponds to the one dimensional algebra: $\mathcal{D}=\mathcal{A}_{0}$
} 
by extending a non-Abelian algebra (with $m=1$ ), so the number of double extensions leading to a given Lie algebra is not unique ${ }^{\text {TI }}$.

Turning to the search of the structure of a Wigner contraction, the only candidates are those enumerated in (2.61). $\mathcal{A}_{3}$ is the Heisenberg algebra, and it is indeed a Wigner contraction of $s o(2,1) \oplus s o(2)$ (which leads to the first possibility in (2.61)). The other candidate is $\mathcal{A}_{6}$, which corresponds to the last possibility in (2.61). To examine this case, we use the further requirement that in a Wigner contraction, $\mathcal{B}$ must be self dual $\square$. For $m=2, \mathcal{B}$ is the two-dimensional, non-Abelian Lie algebra

$$
\left[R_{0}, R_{1}\right]=R_{1}
$$

This algebra is not self-dual, therefore, even if $\mathcal{A}_{6}$ can be obtained by a Wigner contraction, this procedure will not lead to an invariant metric on $\mathcal{A}_{6}$.

\section{Some General Issues Arrising in Gauged WZNW Models Based on Non-Semi-Simple Groups}

Having a family of self-dual algebras, the natural thing to do is to construct the WZNW models based on them. This will be done (for $\mathcal{A}_{3}$ and $\mathcal{A}_{6}$ ) in the next section. However, as in any non-compact Lie-algebra, the invariant metric is not positive definite. In fact, for all the algebras described in section 2 , the metric has more than one negative eigenvalue, therefore the $\sigma$-model obtained from a WZNW model based on them has an unphysical metric - more than one time-like direction - and to correct this we have to gauge out the extra time-like directions. In the proccess of exploring the various possibilities of gauging, we encountered some phenomena that are very common when the algebras involved are not semi-simple. Therefore, before we turn to the consideration of specific models, we describe in this section some of these phenomena and analyse them in a general setting. We start, is subsection 3.1, with a review of the construction of WZNW and gauged WZNW models. In subsection 3.2 we consider situations in which the integration of the gauge fields leads to constraints on the coordinates parametrizing the group manifold. In subsection 3.3 we discuss "singular" gauging, where the restriction of the metric to the gauged subgroup is degenerate. Finaly, in subsection 3.4 we analyze the gauging of a central subgroup.

\footnotetext{
${ }^{15}$ The notion of "depth" of a self-dual Lie algebra, suggested in [15], is still well defined, if one allows only extensions by either a simple or a one-dimensional algebra. Alternatively, the depth can be defined as the minimal number of double extensions.

${ }^{16}$ Actually, the metric is not involved at all in the construction of an algebra by a Wigner contraction (unlike double extension), and all that is needed is a Lie bracket of the form (2.15 2.16). However, we are interested in an algebra with an invariant metric and if we want that this procedure will provide us also with the metric (through (2.21) $), \mathcal{B}$ must be self-dual.
} 


\subsection{The General Setup}

To define a WZNW model [1], one needs a Lie group $G$ and an invariant metric $(\cdot, \cdot)$ on its Lie algebra $d G$. The action of the model is 17

$$
S[g]=\frac{\hbar}{8 \pi}\left[\int_{\Sigma} d^{2} \sigma \sqrt{|h|} h^{\alpha \beta}\left(J_{\alpha}^{L}, J_{\beta}^{L}\right)-\frac{1}{3} \int_{B} d^{3} \sigma \epsilon^{\alpha \beta \gamma}\left(J_{\alpha}^{L},\left[J_{\beta}^{L}, J_{\gamma}^{L}\right]\right)\right]
$$

where the field $g$ is a map from a two dimensional manifold $\Sigma$ to $G, h_{\alpha \beta}$ is a metric on $\Sigma$, and $J^{L}=g^{-1} d g$ is the left invariant form on $G$ taking values in $d G$. In the second term, $B$ is an arbitrary three-dimensional manifold such that $\Sigma$ is its boundary and $g$ is extended arbitrarily from $\Sigma$ to $B$. Choosing a parametrization $x^{\mu}$ for $g$ and substituting it in (3.1) one obtains (at least locally) a $\sigma$-model action (with vanishing dilaton)

$$
\begin{aligned}
S[x] & =\frac{\hbar}{8 \pi} \int_{\Sigma} d^{2} \sigma\left(\sqrt{|h|} h^{\alpha \beta} G_{\mu \nu}(x)+\epsilon^{\alpha \beta} B_{\mu \nu}(x)\right) \partial_{\alpha} x^{\mu} \partial_{\beta} x^{\nu} \\
& =\frac{\hbar}{8 \pi} \int_{\Sigma} d^{2} \sigma\left(\sqrt{|h|} h^{\alpha \beta}+\epsilon^{\alpha \beta}\right) E_{\mu \nu}(x) \partial_{\alpha} x^{\mu} \partial_{\beta} x^{\nu} \quad, \quad E_{\mu \nu} \equiv G_{\mu \nu}+B_{\mu \nu}
\end{aligned}
$$

The WZNW action (3.1) is invariant under the group $G_{L} \otimes G_{R}$, acting in $G$ by

$$
g \rightarrow h_{L} g h_{R}^{-1} \quad, \quad h_{L}, h_{R} \in G
$$

Given a subgroup $H$ of $G_{L} \otimes G_{R}$, one might attempt to gauge it, i.e. to introduce a $d H$-valued gauge field $A$ and to construct an extension $\hat{S}$ of (3.1) that will be invariant under local $H$ transformations

$$
g(\sigma) \rightarrow h_{L}(\sigma) g(\sigma) h_{R}(\sigma)^{-1} \quad, \quad\left(h_{L}(\sigma), h_{R}(\sigma)\right) \in H
$$

Such an extension exists iff $H$ is anomaly free. This criterion can be stated as follows. Let $H_{L, R}$ be the images of $H$ under the natural homomorphisms $G_{L} \otimes G_{R} \rightarrow G_{L, R}$. These homomorphisms define corresponding homomorphisms on the algebras:

$$
d H \rightarrow d H_{L, R} \quad, \quad A \mapsto A^{L, R}
$$

The criterion for gauge invariance is that for any $A_{1}, A_{2} \in d H$

$$
\left(A_{1}^{L}, A_{2}^{L}\right)=\left(A_{1}^{R}, A_{2}^{R}\right) .
$$

\footnotetext{
${ }^{17}$ The coupling $k$ is contained here in the metric.

${ }^{18}$ The invariance of the metric is needed to obtain a representation of the affine Lie algebra. It is less apparent why the metric should be invertible. When the group is simple this question does not arise, since an invariant (non-zero) metric on a simple algebra is always invertible, but this is not true in general. In Appendix A.2 we show that by relaxing the condition of invertibility one does not obtain any new models, therefore, with no loss of generality, we consider only non-degenerate forms.

${ }^{19}$ The conventional coupling constant $\alpha^{\prime}$ is contained here in the background fields $G$ and $B$.
} 
Equivalently one can say that the two metrics induced on $d H$ by (the pullbacks of) (3.5) are the same. Assuming $H$ is indeed anomaly free, the gauge invariant action is [2]

$\hat{S}[g, A]=S[g]+\frac{\hbar}{4 \pi} \int_{\Sigma} d^{2} \sigma\left(\sqrt{|h|} h^{\alpha \beta}+\epsilon^{\alpha \beta}\right)\left[\left(A_{\alpha}^{L}, J_{\beta}^{R}\right)-\left(J_{\alpha}^{L}, A_{\beta}^{R}\right)+\left(A_{\alpha}, A_{\beta}\right)-\left(A_{\alpha}^{L}, g A_{\beta}^{R} g^{-1}\right)\right]$

where $J^{R}=d g g^{-1}$ is the right invariant form on $G$ and $\left(A_{\alpha}, A_{\beta}\right)$ should be understood as $\left(A_{\alpha}^{L}, A_{\beta}^{L}\right)=\left(A_{\alpha}^{R}, A_{\beta}^{R}\right)$. If $h_{\alpha \beta}$ is conformally flat, we can choose light-cone coordinates $\sigma^{ \pm}$, for which the line element on $\Sigma$ is

$$
d s^{2}=2 e^{2 \varphi(\sigma)} d \sigma^{+} d \sigma^{-}
$$

which means that

$$
\sqrt{|h|} h^{\alpha \beta}=\left(\begin{array}{ll}
0 & 1 \\
1 & 0
\end{array}\right) .
$$

In such coordinates (and with $\epsilon^{+-}=1$ ) the action (3.7) takes the simple form

$$
\hat{S}[g, A]=S[g]+\frac{\hbar}{2 \pi} \int_{\Sigma} d^{2} \sigma\left[\left(A_{+}^{L}, J_{-}^{R}\right)-\left(J_{+}^{L}, A_{-}^{R}\right)+\left(A_{+}, A_{-}\right)-\left(A_{+}^{L}, g A_{-}^{R} g^{-1}\right)\right]
$$

To obtain a $\sigma$-model description [21], one integrates out the gauge fields and fixes the gauge. The action is at most quadratic in the gauge fields, therefore, the integration can be performed explicitly. The resulting effective action for $g$ is

$$
\hat{S}_{\mathrm{eff}}[g]=\left.\hat{S}[g, A]\right|_{A=A_{\mathrm{cl}}}+(\text { dilaton term }),
$$

where $A_{\mathrm{cl}}$ is the solution of the classical equations for $A: \delta \hat{S} / \delta A=0$, and the dilaton term originates from the functional determinant which arises in the process of integration [22]. Another possible contribution to the effective action is the trace anomaly [23. When $d H$ is self dual (e.g. Abelian), the adjoint representation of $d H$ is traceless, and there is no trace anomaly. However, when the adjoint representation of $d H$ is not traceless, such a contribution exists and usually leads to a non-local action. When the effective action is local (see also below), it is a $\sigma$-model action

$$
\begin{gathered}
\hat{S}_{\mathrm{eff}}[x]=\frac{\hbar}{8 \pi} \int_{\Sigma} d^{2} \sigma\left[\left(\sqrt{|h|} h^{\alpha \beta} G_{\mu \nu}(x)+\epsilon^{\alpha \beta} B_{\mu \nu}(x)\right) \partial_{\alpha} x^{\mu} \partial_{\beta} x^{\nu}\right. \\
\left.+\sqrt{|h|} R^{(2)} \Phi(x)\right]
\end{gathered}
$$

(where $x^{\mu}$ is an appropriate parametrization for $g$ ) and in light-cone coordinates (where $\left.\sqrt{|h|} R^{(2)}=-8 \partial_{+} \partial_{-} \varphi\right)$, it simplifies to

$$
\hat{S}[x]=\frac{\hbar}{4 \pi} \int_{\Sigma} d^{2} \sigma\left[E_{\mu \nu}(x) \partial_{+} x^{\mu} \partial_{-} x^{\nu}-4 \Phi(x) \partial_{+} \partial_{-} \varphi\right] .
$$

The models presented above are expected to be conformally invariant. When the action is of the $\sigma$-model type, we can verify this to one loop order, by the vanishing of the beta function equations [20]. All the models derived in section 4 passed this check successfully. 


\subsection{The Appearance of Constraints}

To obtain a more explicit expression for $\hat{S}_{\text {eff }}$ in (3.11), let us express $A_{ \pm}$as linear combinations of two distinct bases $\left\{T_{a}^{+}\right\}$and $\left\{T_{a}^{-}\right\}$for $d H: A_{ \pm}=A_{ \pm}^{a} T_{a}^{ \pm}$. Substituting this into (3.10), we obtain

$$
\hat{S}[g, A]=S[g]+\frac{\hbar}{2 \pi} \int_{\Sigma} d^{2} \sigma\left[A_{+}^{a} J_{a}^{-}-J_{b}^{+} A_{-}^{b}+A_{+}^{a} M_{a b} A_{-}^{b}\right]
$$

where

$$
\begin{gathered}
J_{a}^{-}=\left(T_{a}^{L+}, J_{-}^{R}\right) \quad, \quad J_{b}^{+}=\left(J_{+}^{L}, T_{b}^{R-}\right), \\
M_{a b}=\left(T_{a}^{L+}, T_{b}^{L-}-g T_{b}^{R-} g^{-1}\right)=\left(T_{a}^{R+}-g^{-1} T_{a}^{L+} g, T_{b}^{R-}\right)
\end{gathered}
$$

and $\left\{T_{a}^{L \pm}\right\}$ and $\left\{T_{a}^{R \pm}\right\}$ are the images of $\left\{T_{a}^{ \pm}\right\}$in $d H_{L}$ and $d H_{R}$ respectively ${ }^{20}$. The classical equations for $A$ are

$$
M_{a b} A_{-}^{b}+J_{a}^{-}=0 \quad, \quad A_{+}^{a} M_{a b}-J_{b}^{+}=0 .
$$

The matrix $M$ is not invertible in general (more about this later). However, it defines a bilinear form on $d H$ (a rank-2 covariant tensor under a change of basis in $d H$ ) and, therefore, there exists a pair of bases, for which it is diagonal, and in particular assumes the general form

$$
M_{a b}=\left(\begin{array}{cc}
\hat{M}_{\hat{a} \hat{b}} & 0 \\
0 & 0
\end{array}\right),
$$

where $\hat{M}_{\hat{a} \hat{b}}$ is a square and invertible matrix (for a generic choice of $g$ ) and we divided the set $\{a\}$ of indices into two sets $\{\hat{a}\}$ and $\{\check{a}\}$. In these bases the equations (3.17) are equivalent to

$$
A_{-}^{\hat{b}}=-\hat{N}^{\hat{b} \hat{a}} J_{\hat{a}}^{-} \quad, \quad A_{+}^{\hat{a}}=J_{\hat{b}}^{+} \hat{N}^{\hat{b} \hat{a}} .
$$

(where $\left.\hat{N}=\hat{M}^{-1}: \hat{M}_{\hat{a} \hat{b}} \hat{N}^{\hat{b} \hat{c}}=\delta_{\hat{a}}^{\hat{c}}\right)$ and

$$
J_{\breve{a}}^{-}=0 \quad, \quad J_{\breve{b}}^{+}=0 .
$$

\footnotetext{
${ }^{20}$ Note that although these sets span $d H_{L, R}$, they are linearly independent only if the homomorphisms $d H \rightarrow d H_{L, R}$ are injective and we do not assume that it is necessarily so.

${ }^{21} M$ is not symmetric in general, therefore, the diagonalization cannot always be performed with a single basis. However, in some important cases it can. There always exists a (single) basis for which $M$ takes the form

$$
M_{a b}=\left(\begin{array}{cc}
\hat{M}_{\hat{a} \hat{b}} & 0 \\
\check{M}_{\breve{a} \hat{b}} & 0
\end{array}\right)
$$
}

with $\hat{M}_{\hat{a} \hat{b}}$ as above. Therefore, in situations where $M_{a b}=0 \forall g$ implies $M_{b a}=0 \forall g$, we will obtain the form (3.18). This is what happens in "vector" and "axial" gauging: according to (3.16) we have (for any gauge)

$$
M_{a b}=\left(T_{b}^{R}, T_{a}^{R}-g^{-1} T_{a}^{L} g\right)
$$

and for $T_{a}^{L}= \pm T_{a}^{R}$ this implies

$$
M_{a b}(g)=M_{b a}\left(g^{-1}\right) .
$$


Substituting this into (3.14), we obtain

$$
\left.\hat{S}[g, A]\right|_{A=A_{\mathrm{cl}}}=\left\{S[g]+\frac{\hbar}{2 \pi} \int_{\Sigma} d^{2} \sigma\left[J_{\hat{b}}^{+} \hat{N}^{\hat{b} \hat{a}} J_{\hat{a}}^{-}\right]\right\}_{J_{\tilde{a}}^{-}=J_{\tilde{b}}^{+}=0} .
$$

Note that $A_{+}^{\check{a}}$ and $A_{-}^{\check{b}}$ remain undetermined but disappear from the action.

Using an appropriate parametrization $x^{\mu}$ for $g$, one obtains an action of the form

$$
\hat{S}_{\mathrm{eff}}[x]=\frac{\hbar}{4 \pi} \int_{\Sigma} d^{2} \sigma\left[E_{\mu \nu}(x) \partial_{+} x^{\mu} \partial_{-} x^{\nu}-4 \Phi(x) \partial_{+} \partial_{-} \varphi\right]_{J_{\breve{a}}^{-}=J_{\breve{b}}^{+}=0} .
$$

where the dilaton background field is

$$
\begin{aligned}
\Phi(x)= & -\frac{1}{2} \log |\operatorname{det} \hat{M}|+\text { const. } \\
& +\left(\text { contributions from the delta functions } \delta\left(J_{\breve{a}}^{-}\right), \delta\left(J_{\breve{b}}^{+}\right)\right) .
\end{aligned}
$$

This looks almost as a $\sigma$-model action, but one must still fix the gauge and implement the constraints. When there are no constraints, this indeed leads to a $\sigma$-model action, but, since the constraints are not algebraic, their implementation may lead to a more complicated (e.g. non local) action 22 .

\subsection{Singular Gauging and Extended Gauge Invariance}

By "singular gauging" we mean gauging a subgroup $H$ such that the metric induced on its algebra $d H$ is degenerate. This includes the extreme case of "null gauging", for which the metric vanishes completely. Consider first this last case. The term $\left(A_{+}, A_{-}\right)$vanishes identically, therefore, $A_{-}^{L}$ and $A_{+}^{R}$ do not appear in the action (3.10). This means that the action is determined only by $H_{L}$ and $H_{R}$ and there is no trace of the particular choice ${ }^{23}$ of a subgroup $H$ of $H_{L} \otimes H_{R}$. In particular, the action coincides with the action obtained by gauging the whole $H_{L} \otimes H_{R}$ group. This has the following important implications:

1. The gauge symmetry group of the resulting model is $H_{L} \otimes H_{R}$ and is typically larger than the group $H$ we intended to gauge (the group for which we introduced gauge fields). To obtain a $\sigma$-model, one has to fix this whole extended gauge freedom. This explains why null gauging usually reduces the dimension of the $\sigma$-model target manifold more then by $\operatorname{dim} H$ (for $H_{L}$ and $H_{R}$ isomorphic to $H$ it reduces by $2 \operatorname{dim} H$ - this was observed already in [19] in a specific example).

\footnotetext{
${ }^{22}$ In this context it is interesting to know when the constraints appear. In Appendix A.3 we show that when an appropriate single "diagonalizing" basis $T_{a}^{+}=T_{a}^{-}$for $d H$ exists (and in particular for axial and vector gauging), constraints can appear only if $G$ is not semi-simple or the gauging is singular (i.e. the metric $(\cdot, \cdot)_{d H}$ induced on $d H$ is degenerate).

${ }^{23}$ The gauge field has the following general form

$$
A_{ \pm}^{L, R}=A_{ \pm}^{a} T_{a}^{L, R}
$$

and $H$ is determined by the relation between $T_{a}^{L}$ and $T_{a}^{R}$, which manifest itself by the dependence between $A_{+}^{L}$ and $A_{+}^{R}$ and similarly between $A_{-}^{L}$ and $A_{-}^{R}$.
} 
2. The vanishing of the metric on $d H$, guaranties that the anomaly condition (3.6) is satisfied for each choice of a subgroup $H$ of $\hat{H}=H_{L} \otimes H_{R}$ and, therefore, one might expect that in this case the variety of possible models is considerably larger. Contrary to this expectation, we found that all these potential models coincide $\underbrace{2} \mathrm{~F}^{2}$.

Returning to the general case, we define

$$
\mathcal{J} \equiv\{R \in d H \mid(R, T)=0, \forall T \in d H\}
$$

The invariance of the metric implies that $\mathcal{J}$ is an ideal in $d H$, therefore, it corresponds to a normal subgroup $N$ of $H: \mathcal{J}=d N$. Taking for an element of $H$ a parametrization of the form $h(x, y)=n(x) k(y)$, where $n \in N$ and $k$ parametrizes elements of the quotient group, $K \equiv H / N$, the action of $H$ in $G$ is

$$
g \rightarrow\left[n_{L}(x) k_{L}(y)\right] g\left[k_{R}^{-1}(y) n_{R}^{-1}(x)\right],
$$

however, similar arguments to those presented above imply that the action is actually invariant also under local $N_{L} \otimes N_{R}$, i.e. $N$ acts independently from the left and from the right, so the action of the full gauge group is:

$$
g \rightarrow\left[n_{L}\left(x_{L}\right) k_{L}(y)\right] g\left[k_{R}^{-1}(y) n_{R}^{-1}\left(x_{R}\right)\right]
$$

(observe that this is indeed a group, i.e. closed under composition, because $N$ is a normal subgroup of $H$ ).

\subsection{Gauging a Central Subgroup}

Gauging a central subgroup (i.e. taking $H_{L}$ and $H_{R}$ that commute with all elements of $G$ ) is expected to be a relatively simple choice of gauging. However, in many cases such a choice does not lead to a new $\sigma$-model backgrounds. In particular, we show here that when $H_{L}=H_{R}$, the resulting model is an (ungauged) WZNW model[O. Denotinger $\bar{H}=H_{L}=H_{R}$, the model is based on the group $K / \bar{H}_{0}$, where $K$ is the subgroup of $G$ generated by all the generators of $G$ that are orthogonal to $d \bar{H}$, and $\bar{H}_{0}$ is the subgroup of $\bar{H}$ generated by all the "null" generators: $d \bar{H}_{0}=d \bar{H} \cap d K$.

First we show that those generators of $G$ that are in $d \bar{H}$ but not in $d \bar{H}_{0}$ do not contribute to the final gauged action. The restriction of the metric on $d G$ to $d \bar{H}$, being a symmetric bilinear form on $d \bar{H}$, can be diagonalized, therefore, if it does not vanish, we

\footnotetext{
${ }^{24}$ For $H_{L}=H_{R}$ Abelian, this is a trivial realization of axial-vector duality [24].

${ }^{25}$ Note that this result holds also when $H$ is central (i.e. commutes with all elements of $G$ ), in spite of the fact that the diagonal ("vector") subgroup of $C_{L} \otimes C_{R}$, for $C$ central, acts trivially in $G$ and, therefore, cannot be gauged in the usual sense. This point is explained in Appendix A.4.

${ }^{26}$ In Appendix A.5 we analyze the $H_{L} \neq H_{R}$ case for one dimensional $H$. We also comment on the $H_{L} \neq H_{R}$ case of null $H$ in a footnote after eq. (3.37). These cases also do not lead to new $\sigma$-model backgrounds.

${ }^{27}$ We note the distinction between $\bar{H} \subset G$ and $H \subset H_{L} \otimes H_{R} \subset G_{L} \otimes G_{R}$.
} 
have a decomposition $d \bar{H}=d \bar{H}_{0} \oplus d \bar{H}_{1}$, such that $\left(d \bar{H}, d \bar{H}_{0}\right)=0$ and the restriction of the metric to $d \bar{H}_{1}$ is non-degenerate. Using the invariance of the metric on $d G$, we have

$$
\left([d G, d G], d \bar{H}_{1}\right)=\left(d G,\left[d G, d \bar{H}_{1}\right]\right)=0
$$

(since $\bar{H}$ is central), which implies that $[d G, d G]$ is contained in $d \bar{H}_{1}^{\perp}$ and, in particular, that $d \bar{H}_{1}^{\perp}$ is a subalgebra of $d G$ (in fact it is an ideal, since it commutes with $d \bar{H}_{1}$ ). Let $G_{0}$ be the corresponding subgroup of $G:\left(d \bar{H}_{1}\right)^{\perp}=d G_{0}$. The metric on $d \bar{H}_{1}$ is non-degenerate, therefore,

$$
d G_{0} \cap d \bar{H}_{1}=\{0\},
$$

so $d G$ is a direct sum of orthogonal ideals

$$
d G=d G_{0} \oplus d \bar{H}_{1} \quad, \quad\left(d G_{0}, d \bar{H}_{1}\right)=0 .
$$

The action of a WZNW model based on a direct product of groups $G=G_{0} \otimes G_{1}$, where the algebras of the groups are orthogonal to each other, decomposes to a sum of independent terms, a term for each factor. When the gauged group is also a direct product of the form

$$
H=H_{0} \otimes H_{1} \quad, \quad H_{i} \subset\left(G_{i}\right)_{L} \otimes\left(G_{i}\right)_{R} \quad, \quad(i=0,1)
$$

the decomposition of the action holds also in the gauged model. However, since $\bar{H}_{0}$ is null, (3.31) always holds. To show this, let us construct an appropriate basis for $d H$. Since $d H \subset d H_{L} \oplus d H_{R}$, a basis element is represented by a pair $\left(\left(T_{i}^{L}\right)_{0}+\left(T_{i}^{L}\right)_{1},\left(T_{i}^{R}\right)_{0}+\left(T_{i}^{R}\right)_{1}\right)$. According to the general discussion of null gauging, $d H_{0}=d\left(H_{0}\right)_{L} \oplus d\left(H_{0}\right)_{R}$, so we can choose a basis $($ for $d H)$ that includes a basis for $d\left(H_{0}\right)_{L}\left(\right.$ i.e. $\left.\left.\left(T_{i}^{L}\right)_{1}=\left(T_{i}^{R}\right)_{0}=\left(T_{i}^{R}\right)_{1}\right)=0\right)$ and a basis for $d\left(H_{0}\right)_{R}$. With such a basis we can set $\left(T_{i}^{L}\right)_{0}=\left(T_{i}^{R}\right)_{0}=0$ whenever either $\left(T_{i}^{L}\right)_{1} \neq 0$ or $\left(T_{i}^{R}\right)_{1} \neq 0$ and the resulting set will remain a basis. This means that we indeed have the direct product structure (3.31) ${ }^{28}$, with $G_{1}=\bar{H}_{1}$ and we can analyze each factor separately. The $G_{1}$ part corresponds to $G$ Abelian, $H_{L}=H_{R}=G$, and its contribution to the action vanishes (more about this in Appendix A.4). This enables us (up to topological issues) to restrict our attension to the $G_{0}$ part of $G$ and the $H_{0}$ part of $H$, i.e. , to consider null $H$.

According to (3.28),

$$
[d G, d G] \subset d \bar{H}^{\perp}
$$

where $d \bar{H}^{\perp}$ is the orthogonal complement of $d \bar{H}$. This implies that $d \bar{H}^{\perp}$ is a subalgebra. We will denote by $K$ the corresponding subgroup of $G$. Note that $\bar{H} \subset K$, since $d \bar{H}$ is null. To construct a parametrization of $G$ we chose a subspace $\mathcal{B}$ of $d G$ such that $d G=\mathcal{B} \oplus d K$ (direct sum of vector spaces) and a basis $\left\{S_{a}\right\}_{a=1}^{m}$ for $\mathcal{B}(m=\operatorname{dim} \mathcal{B}=\operatorname{dim} \bar{H})$. We use (3.32) again, to deduce that $d G$ has the following structure

$$
d G=\mathbb{R} S_{1} \in\left(\mathbb{R} S_{2} \in\left(\ldots \in\left(\mathbb{R} S_{m} \in d K\right) \ldots\right)\right)
$$

\footnotetext{
${ }^{28}$ This result depends on the assumption $H_{L}=H_{R}$. See Appendix A.5 for a counter-example in the general case.

29 Recall that we denote by $\mathcal{B} \oplus \mathcal{J}$ a semi-direct sum of algebras $\mathcal{B}$ and $\mathcal{J}$, in which $\mathcal{J}$ is an ideal. Similarly, $H \otimes N$ denotes a semi-direct product of groups $H$ and $N$, in which $N$ is a normal subgroup.
} 
(where $\mathbb{R} S_{a} \equiv \operatorname{sp}_{\mathbb{R}}\left\{S_{a}\right\}$ ) and, therefore

$$
G=e^{\mathbb{R} S_{1}} \otimes\left(e^{\mathbb{R} S_{2}} \otimes\left(\ldots \otimes\left(e^{\mathbb{R} S_{m}} \otimes K\right) \ldots\right)\right)
$$

(where $\exp \left(\mathbb{R} S_{a}\right)$ denotes the one-parameter-group generated by $S_{a}$ ). This suggest a parametrization for $g \in G$ of the form

$$
g(x, y, z)=e^{y^{m} S_{m}} \ldots e^{y^{1} S_{1}} k(x) e^{z^{a} T_{a}}
$$

where $\left\{T_{a}\right\}_{a=1}^{m}$ is a basis for $\bar{H}$ and $k(x)$ is some parametrization of the quotient group $K / \bar{H}$. In these coordinates, the invariant form $J^{L}$ takes the form (using (4.6))

$$
\begin{aligned}
J^{L} \equiv g^{-1} d g= & T_{a} d z^{a}+k^{-1} d k+k^{-1}\left[S_{1} d y^{1}+e^{-y^{1} \operatorname{ad}\left(S_{1}\right)}\left(S_{2} d y^{2}+\right.\right. \\
& \left.\left.+e^{-y^{2} \operatorname{ad}\left(S_{2}\right)}\left(\ldots\left(S_{m-1} d y^{m-1}+e^{-y^{m-1} \operatorname{ad}\left(S_{m-1}\right)} S_{m} d y^{m}\right) \ldots\right)\right)\right] k \\
= & T_{a} d z^{a}+S_{a} d y^{a}+J_{\perp}^{L}(x, y) \quad, \quad J_{\perp}^{L} \in d K
\end{aligned}
$$

(the last equality follows from $e^{S} T e^{-S}-T \in[d G, d G] \subset d K$ ), so the gauged WZNW action is of the form

$$
\hat{S}[g, A]=S[g]+\frac{\hbar}{2 \pi} \int_{\Sigma} d^{2} \sigma\left[\left(A_{+}^{L}, S_{a}\right) \partial_{-} y^{a}-\left(A_{-}^{R}, S_{a}\right) \partial_{+} y^{a}\right] .
$$

The integration over $A$ yields the constraints

$$
\partial_{-} y^{a}=0 \quad \text { for components coupled to } d H_{L} \text {; }
$$$$
\partial_{+} y^{a}=0 \quad \text { for components coupled to } d H_{R} \text {. }
$$

which means, since we assume ${ }^{\beta 1} H_{L}=H_{R}$, that $d y^{a}=0 \forall a . J^{L}$ simplifies to

$$
J^{L}=T_{a} d z^{a}+g_{0}^{-1} d g_{0} \in d G_{0},
$$

the $z$ dependence disappear from the action (because $T_{a}$ is central and orthogonal to $d G_{0}$ ) and the resulting effective action coincides with the WZNW action for the group $K / \bar{H}$ (with the target space variables $x^{i}$ ) 32 .

\footnotetext{
${ }^{30}$ For some Abelian subalgebra of $d G$ contained in $\mathcal{B}$, the corresponding subgroup of $G$ may be compact. In this case the lefthand side of (3.34) should be divided by a discrete group. This is irrelevant to the subsequent discussion and, therefore, will be ignored.

${ }^{31}$ For $d H_{L} \neq d H_{R}$ we may define

$$
\bar{H}=H_{L} H_{R} \equiv\left\{h_{L} h_{R} \mid h_{L, R} \in H_{L, R}\right\}
$$

which is also a central subgroup. If $\bar{H}$ is null, the whole derivation up to this point is valid. $A_{+}^{L}$ and $A_{-}^{R}$ in eq. (3.37) are components of two different gauge fields, corresponding to left and right translations, respectively. Since $d H_{L} \neq d H_{R}$, some components of $y$ will be constrained to depend on one of the light-cone coordinates and it is not clear if the resulting effective action can be brought to the form of a $\sigma$-model.
}

${ }^{32}$ Note that all the non-invariant fields disappear from the action so no gauge fixing is needed. 
To summarize, when $H_{L}=H_{R}$ is central, there is always an (orthogonal) direct product decomposition

$$
\begin{gathered}
G=G_{0} \otimes G_{1} \quad, \quad d G_{0} \perp d G_{1}, \\
H=H_{0} \otimes H_{1} \quad, \quad\left(H_{0}\right)_{L}=\left(H_{0}\right)_{R} \subset G_{0} \quad, \quad\left(H_{1}\right)_{L}=\left(H_{1}\right)_{R}=G_{1}
\end{gathered}
$$

such that $G_{1}$ is central and $H_{0}$ is null. Denoting $\bar{H}_{0} \equiv\left(H_{0}\right)_{L}=\left(H_{0}\right)_{R}$ and $d K=\left(d \bar{H}_{0}\right)^{\perp}$ we have

$$
G_{0} \supset K \supset \bar{H}_{0}
$$

and the resulting action is the action of a WZNW model for the group $K / \bar{H}_{0}$.

\section{WZNW and gauged WZNW models based on $\mathcal{A}_{n}$}

In this section we will present some (gauged and ungauged) WZNW models based on the algebras $\mathcal{A}_{3}$ and $\mathcal{A}_{6}$. The corresponding $\sigma$-model background fields, and some related tensors, are listed in Appendix B. For all the $\sigma$-models obtained, the one-loop beta functions [20] vanish and the central charge is equal to the dimension of the $\sigma$-model target manifold.

For the construction of a WZNW model, one needs a convenient parametrization of the corresponding group. For a compact group $G$ one often chooses the parametrization $g=\exp \left(x^{a} T_{a}\right)$, where $\left\{T_{a}\right\}$ is some basis for $d G$. However, in general the exponential map exp: $d G \rightarrow G$ is not onto, even if $G$ is connected ${ }^{35}$ and when it is not, a different parametrization is needed. For solvable Lie algebras (as the algebras $\mathcal{A}_{n}$ ), one can exploit the fact [25] that any such algebra is a repeated semi-direct sum of one-dimensional Lie algebras and consequently [25] the corresponding (connected and simply connected covering) group is a semi-direct product of one-dimensional groups. This is the approach we use. The grading on $\mathcal{A}_{n}$ implies that

$$
\mathcal{A}_{n}=\mathbb{R} T_{0} \oplus\left(\mathbb{R} T_{1} \oplus\left(\ldots \in\left(\mathbb{R} T_{n-1} \in \mathbb{R} T_{n}\right) \ldots\right)\right)
$$

so the simply connected covering group of $\mathcal{A}_{n}$ is

$$
G_{n}=e^{\mathbb{R} T_{0}} \otimes\left(e^{\mathbb{R} T_{1}} \otimes\left(\ldots \otimes\left(e^{\mathbb{R} T_{n-1}} \otimes e^{\mathbb{R} T_{n}}\right) \ldots\right)\right) .
$$

This means that the map

$$
\left(x_{0}, \ldots, x_{n}\right) \rightarrow g=e^{x_{n} T_{n}} \ldots e^{x_{0} T_{0}}
$$

${ }^{33}$ For example, it can be shown that an $S L(2, \mathbb{R})$ matrix of the form

$$
\left(\begin{array}{cc}
-1 & \beta \\
0 & -1
\end{array}\right) \quad, \quad \beta \neq 0
$$

is not in the range of the exponential map. 
is a homeomorphism from $\mathbb{R}^{n+1}$ onto $G_{n}$. This is, therefore, a suitable parametrization.

Next we derive expressions for the invariant forms on $G_{n}$. Denoting by $\operatorname{ad}(S)$ the adjoint action of $S \in d G$ :

$$
\operatorname{ad}(S): T \rightarrow[S, T] \quad, \quad S, T \in d G
$$

and

$$
e^{x \operatorname{ad}(S)} \equiv \sum_{k=0}^{\infty} \frac{x^{k}}{k !} \operatorname{ad}(S)^{k}
$$

we have

$$
e^{S} T e^{-S}=e^{\operatorname{ad}(S)}(T)
$$

Using this formula we obtain:

$$
\begin{aligned}
J^{L} & \equiv g^{-1} d g \\
& =T_{0} d x_{0}+e^{-x_{0} \operatorname{ad}\left(T_{0}\right)}\left(T_{1} d x_{1}+e^{-x_{1} \operatorname{ad}\left(T_{1}\right)}\left(\ldots\left(T_{n-1} d x_{n-1}+e^{-x_{n-1} \operatorname{ad}\left(T_{n-1}\right)} T_{n} d x_{n}\right) \ldots\right)\right), \\
J^{R} & \equiv d g g^{-1} \\
& =T_{n} d x_{n}+e^{x_{n} \operatorname{ad}\left(T_{n}\right)}\left(T_{n-1} d x_{n-1}+e^{x_{n-1} \operatorname{ad}\left(T_{n-1}\right)}\left(\ldots\left(T_{1} d x_{1}+e^{x_{1} \operatorname{ad}\left(T_{1}\right)} T_{0} d x_{0}\right) \ldots\right)\right) .
\end{aligned}
$$

$T_{0}$ acts by multiplication:

$$
e^{x_{0} \operatorname{ad}\left(T_{0}\right)}: T_{i} \rightarrow e^{-\hat{i} x_{0}} T_{i}
$$

and all other generators are nilpotent, which means that the sum in (4.5) is finite, therefore, formulas (4.7) and (4.8) provide a well defined algorithm for the computation of the invariant forms.

\subsection{Models based on $\mathcal{A}_{3}$}

\subsubsection{The ungauged model}

Using the parametrization (4.3) and the formulas (4.7) and (4.8) with $n=3$ we obtain

$$
\begin{aligned}
J^{L}= & T_{0} d x_{0}+e^{x_{0}} T_{1} d x_{1}+e^{-x_{0}} T_{2} d x_{2}+T_{3}\left(x_{1} d x_{2}+d x_{3}\right) \\
J^{R}= & T_{0} d x_{0}+T_{1}\left(d x_{1}+x_{1} d x_{0}\right)+T_{2}\left(d x_{2}-x_{2} d x_{0}\right) \\
& +T_{3}\left(d x_{3}+x_{2} d x_{1}+x_{2} x_{1} d x_{0}\right)
\end{aligned}
$$

Substituting these expressions in the WZNW action (3.1), together with the Lie bracket (1.1) and the invariant metric

$$
\left(T_{i}, T_{j}\right)=\delta_{i+j-3}+b \delta_{i} \delta_{j}
$$

\footnotetext{
${ }^{34}$ This metric is obtained from the diagonal one (with $b=0$ ) by the (automorphic) change of basis $T_{0} \rightarrow T_{0}+\frac{1}{2} b T_{3}$, therefore, keeping $b$ arbitrary will provide us with a convenient way of performing a family of gaugings.

${ }^{35}$ We could take a constant multiple of (4.12). This is equivalent to changing the $\sigma$-model coupling constant $\alpha^{\prime}$, i.e. rescaling the background fields $\left(G_{\mu \nu}\right.$ and $B_{\mu \nu}$ but not $\left.\Phi\right)$.
} 
one obtains the following $\sigma$-model action:

$$
S=\frac{\hbar}{4 \pi} \int_{\Sigma} d^{2} \sigma\left[\partial_{+} x_{0}\left(2 \partial_{-} x_{3}+b \partial_{-} x_{0}\right)+2 \partial_{+} x_{2}\left(\partial_{-} x_{1}+x_{1} \partial_{-} x_{0}\right)\right] .
$$

This is an analytic continuation of the model in [3].

\subsubsection{Gauging $T_{0}$}

Next we gauge the symmetry

$$
g \rightarrow h_{L} g h_{R}^{-1}, \quad h_{L, R}=e^{\theta_{L, R} T_{0}}
$$

which, in the coordinates (4.3) takes the form

$$
\begin{aligned}
& x_{0} \rightarrow x_{0}+\theta_{L}-\theta_{R} \\
& x_{1} \rightarrow e^{-\theta_{L}} x_{1} \\
& x_{2} \rightarrow e^{\theta_{L}} x_{2} \\
& x_{3} \rightarrow x_{3} .
\end{aligned}
$$

Since $\left(T_{0}, T_{0}\right)=b$, for $b \neq 0$ the anomaly condition is $\theta_{L}= \pm \theta_{R}$ ("vector/axial" gauging), while for $b=0$ the gauging is null, therefore, anomaly-free and, by the general discussion in section 3.3, independent of the relation between $\theta_{L}$ and $\theta_{R}$. Hence we can restrict attention to the cases $\theta_{L}= \pm \theta_{R}$. Using the general notation introduced in section 3.2, we have

$$
M \equiv\left(T_{0}, T_{0} \mp g T_{0} g^{-1}\right)=b(1 \mp 1) \mp x_{1} x_{2}
$$

(since $g T_{0} g^{-1}=T_{0}+x_{1} T_{1}-x_{2} T_{2}+x_{1} x_{2} T_{3}$ ),

$$
J^{+}=\left(J_{+}^{L}, \pm T_{0}\right)= \pm\left(b \partial_{+} x_{0}+x_{1} \partial_{+} x_{2}+\partial_{+} x_{3}\right)
$$

and

$$
J^{-}=\left(T_{0}, J_{-}^{R}\right)=\left(b+x_{1} x_{2}\right) \partial_{-} x_{0}+x_{2} \partial_{-} x_{1}+\partial_{-} x_{3}
$$

$M$ does not vanish identically (not even for $b=0$ ), so the effect of the gauging is to add to the Lagrangian the contribution

$$
\frac{\hbar}{2 \pi}\left[\frac{1}{M} J^{+} J^{-}+(\log M) \partial_{+} \partial_{-} \varphi\right]
$$

To proceed, we need a gauge choice. In the case of axial gauging $\theta_{L}=-\theta_{R}$, we can fix $x_{0}=0$ and obtain

$$
\begin{gathered}
\hat{S}_{\mathrm{eff}}\left(x_{1}, x_{2}, x_{3}\right)=\frac{\hbar}{4 \pi} \int_{\Sigma} d^{2} \sigma\left[2 \partial_{+} x_{1} \partial_{-} x_{2}-2 p\left(x_{1} \partial_{+} x_{2}+\partial_{+} x_{3}\right)\left(x_{2} \partial_{-} x_{1}+\partial_{-} x_{3}\right)\right. \\
\left.+2 \log \left|x_{1} x_{2}+2 b\right| \partial_{+} \partial_{-} \varphi\right]
\end{gathered}
$$


with

$$
p=\frac{1}{x_{1} x_{2}+2 b} .
$$

For vector gauging $\theta_{L}=\theta_{R}, x_{0}$ is invariant and the symmetry acts only on $x_{1}$ and $x_{2}$. This action does not alter the sign so strictly speaking one cannot fix a coordinate to a constant. However, at $x_{1} x_{2}=0$ there is a singularity, so non-singular field configurations will have coordinates with a homogeneous sign and the field configuration space is divided to sectors. Moreover, the action is invariant ${ }^{30}$ under $x_{i} \rightarrow-x_{i}, i=1,2$ so we can restrict ourselves to the $x_{1}>0$ sector. Therefore, we can choose the gauge $x_{1}=1$ and the result is

$$
\begin{aligned}
\hat{S}_{\mathrm{eff}}\left(x_{0}, x_{2}, x_{3}\right)= & \frac{\hbar}{4 \pi} \int_{\Sigma} d^{2} \sigma\left[\partial_{+} x_{0} \partial_{-}\left(b x_{0}+2 x_{2}+2 x_{3}\right)-\right. \\
& \left.-\frac{2}{x_{2}} \partial_{+}\left(b x_{0}+x_{2}+x_{3}\right)\left(\left(b+x_{2}\right) \partial_{-} x_{0}+\partial_{-} x_{3}\right)+2 \log \left|x_{2}\right| \partial_{+} \partial_{-} \varphi\right] .
\end{aligned}
$$

For $b=0$ the two models in eqs. (4.19) and (4.21) are apparently different, although they should be the same according to the general discussion in section 3.3. But this is the result of a different gauge choice. In fact the gauge choice $x_{1}=1$ is equally valid for the axial gauging when $b=0$ and it leads to identical models. The metric in eqs. (4.19) and (4.21) with $b=0$ is degenerate (see Appendix B). This is expected, since $b=0$ corresponds to a null gauging, and the degeneracy is the result of the extended gauge symmetry, as discussed in section 3.3. Indeed, the independence of $\theta_{L}$ and $\theta_{R}$ allows the fixing of both $x_{0}$ and $x_{1}$. The action obtained is

$$
\hat{S}_{\mathrm{eff}}\left(x_{2}, x_{3}\right)=\frac{\hbar}{4 \pi} \int_{\Sigma} d^{2} \sigma\left[-\frac{2}{x_{2}} \partial_{+}\left(x_{2}+x_{3}\right) \partial_{-} x_{3}+2 \log \left|x_{2}\right| \partial_{+} \partial_{-} \varphi\right]
$$

and the corresponding metric is non-degenerate.

\subsection{Models based on $\mathcal{A}_{6}$}

\subsubsection{The ungauged model}

Using the parametrization (4.3) and the formulas (4.7) and (4.8) with $n=6$ we obtain

$$
\begin{aligned}
J^{L}= & T_{0} d x_{0}+T_{1} e^{x_{0}} d x_{1}+T_{2} e^{-x_{0}} d x_{2}+T_{3}\left(d x_{3}+x_{1} d x_{2}\right) \\
& +T_{4} e^{x_{0}}\left(d x_{4}-x_{1} d x_{3}-\frac{1}{2} x_{1}^{2} d x_{2}\right)+T_{5} e^{-x_{0}}\left(d x_{5}+x_{2} d x_{3}\right) \\
& +T_{6}\left(d x_{6}+x_{1} d x_{5}-x_{2} d x_{4}+x_{1} x_{2} d x_{3}\right)
\end{aligned}
$$

and

$$
\begin{aligned}
J^{R}= & T_{0} d x_{0}+T_{1}\left(d x_{1}+x_{1} d x_{0}\right)+T_{2}\left(d x_{2}-x_{2} d x_{0}\right) \\
& +T_{3}\left(d x_{3}+x_{2}\left(d x_{1}+x_{1} d x_{0}\right)\right)+T_{4}\left(d x_{4}-x_{3} d x_{1}+\left(x_{4}-x_{3} x_{1}\right) d x_{0}\right) \\
& +T_{5}\left(d x_{5}+x_{3} d x_{2}-\frac{1}{2} x_{2}^{2} d x_{1}-\left(x_{5}+x_{3} x_{2}+\frac{1}{2} x_{2}^{2} x_{1}\right) d x_{0}\right) \\
& +T_{6}\left(d x_{6}-x_{4} d x_{2}+x_{5} d x_{1}+\left(x_{5} x_{1}+x_{4} x_{2}\right) d x_{0}\right) .
\end{aligned}
$$

\footnotetext{
${ }^{36}$ This invariance originates from the fact that the map $T_{i} \rightarrow-T_{i}, i=1,2$, is an isometric automorphism in $\mathcal{A}_{3}$, i.e. it conserves the metric and the Lie bracket.
} 
Substituting these expressions in the WZNW action (3.1), together with the Lie bracket (1.1) and the metric

$$
\left(T_{i}, T_{j}\right)=\delta_{i+j-6}+b \delta_{i} \delta_{j}
$$

one obtains the following $\sigma$-model action:

$$
\begin{aligned}
S=\frac{\hbar}{4 \pi} \int_{\Sigma} d^{2} \sigma[ & \sum_{i=0}^{6} \partial_{+} x_{i} \partial_{-} x_{6-i}+b \partial_{+} x_{0} \partial_{-} x_{0}+ \\
& +x_{1}\left(\partial_{+} x_{2} \partial_{-} x_{3}-\partial_{+} x_{3} \partial_{-} x_{2}+2 \partial_{+} x_{5} \partial_{-} x_{0}\right) \\
& \left.+x_{2}\left(\partial_{+} x_{1} \partial_{-} x_{3}+\partial_{+} x_{3} \partial_{-} x_{1}-2 \partial_{+} x_{4} \partial_{-} x_{0}\right)+2 x_{1} x_{2} \partial_{+} x_{3} \partial_{-} x_{0}\right]
\end{aligned}
$$

\subsubsection{Gauging the Center}

The simplest way to obtain a model with a reduced dimension is to gauge the center of $G_{6}$, which is the one-parameter group generated by $T_{6}$. This will also serve as an illustration of the general discussion in section 3.4. More precisely, we gauge the twodimensional subgroup $H$ of $G_{L} \otimes G_{R}$ whose action in $G$ is

$$
g \rightarrow h_{L} g h_{R}^{-1}, \quad h_{L, R}=e^{\theta_{L, R} T_{6}}
$$

(in the parametrization (4.3), this is $x_{6} \rightarrow x_{6}+\theta_{L}-\theta_{R}$ ). This gauging is anomaly-free for independent $\theta_{L}$ and $\theta_{R}$ because $T_{6}$ is null in the metric (4.25). The resulting action is

$$
\hat{S}[g, A]=S[g]+\frac{\hbar}{2 \pi} \int_{\Sigma} d^{2} \sigma\left(A_{+} \partial_{-}-A_{-} \partial_{+}\right) x_{0}
$$

Integrating out the gauge fields results with the constraint $x_{0}=$ const. . Imposing the constraint in the action $\hat{S}$ eliminates the $x_{0}$ and $x_{6}$ dependence and we obtain a 5 -dimensional (gauge-invariant) $\sigma$-model action:

$$
\begin{aligned}
& \hat{S}_{\text {eff }}\left(x_{1}, x_{2}, x_{3}, x_{4}, x_{5}\right)=\left.S\right|_{d x_{0}=0} \\
& =\frac{\hbar}{4 \pi} \int_{\Sigma} d^{2} \sigma\left[\sum_{i=1}^{5} \partial_{+} x_{i} \partial_{-} x_{6-i}+x_{1}\left(\partial_{+} x_{2} \partial_{-} x_{3}-\partial_{+} x_{3} \partial_{-} x_{2}\right)\right. \\
& \left.\quad+x_{2}\left(\partial_{+} x_{1} \partial_{-} x_{3}+\partial_{+} x_{3} \partial_{-} x_{1}\right)\right] .
\end{aligned}
$$

which is the WZNW action for the group whose algebra is 59

$$
\mathcal{A}=\operatorname{sp}\left\{T_{1}, \ldots, T_{6}\right\} / \operatorname{sp}\left\{T_{6}\right\}
$$

\footnotetext{
${ }^{37}$ Recall that $G_{6}$ is the simply connected covering group of $\mathcal{A}_{6}$, as defined in eq. (4.2).

${ }^{38} \mathrm{As}$ explained in the general discussion, this is equivalent to vector/axial gauging of a one dimensional subgroup, which in our notation corresponds to $\theta_{L}= \pm \theta_{R}$.

${ }^{39}$ This is the unique 5-dimensional self-dual Lie algebra, appearing in the list of [12].
} 


\subsubsection{Towards a Four Dimensional Model}

To get down to 4 dimensions, we must add another generator to $H$. We want to explore as many options as possible, so we take the action of $H$ in $G$ to be

$$
g \rightarrow h_{L} g h_{R}^{-1} \quad, \quad h_{L, R}=e^{\theta_{L, R} T_{6}+\varphi_{L, R} T_{m}}
$$

with $T_{m}=a_{2} T_{2}+a_{4} T_{4}$, where $a_{2,4}$ are parameters which determine the choice of the additional generator. Since

$$
\left(T_{m}, T_{6}\right)=0 \quad, \quad\left(T_{m}, T_{m}\right)=2 a_{2} a_{4},
$$

for $a_{2} a_{4} \neq 0$ the anomaly condition is $\varphi_{L}= \pm \varphi_{R}$ ("vector/axial" gauging), while for $a_{2} a_{4}=0\left(T_{m}\right.$ proportional to $T_{2}$ or $\left.T_{4}\right)$ the gauging is null and, therefore, anomaly-free for any $\varphi_{L, R}$. To fit to the general notation introduced in section (3.1), we choose a (single) basis for $d H$ with

$$
\begin{gathered}
\left\{T_{a}^{L}\right\}=\left\{\alpha_{L} T_{m}, T_{6}\right\} \quad, \quad\left\{T_{a}^{R}\right\}=\left\{\alpha_{R} T_{m}, T_{6}\right\} . \\
g T_{m} g^{-1}=a_{2} e^{x_{0}}\left(T_{2}-x_{1} T_{3}+\left(x_{1} x_{2}+x_{3}\right) T_{5}\right) \\
+\left(a_{4} e^{-x_{0}}-\frac{1}{2} a_{2} e^{x_{0}} x_{1}^{2}\right) T_{4}+\left[a_{4} x_{2} e^{-x_{0}}-a_{2} e^{x_{0}}\left(x_{4}+\frac{1}{2} x_{1}^{2} x_{2}\right)\right] T_{6},
\end{gathered}
$$

therefore,

$$
M_{a b} \equiv\left(T_{a}^{L}, T_{b}^{L}-g T_{b}^{R} g^{-1}\right)=\left(\begin{array}{cc}
M & 0 \\
0 & 0
\end{array}\right)
$$

with

$$
M=\frac{1}{2} a_{2}^{2} \alpha_{L} \alpha_{R} e^{x_{0}} x_{1}^{2}+2 a_{2} a_{4}\left(\alpha^{2}-\alpha_{L} \alpha_{R} \cosh x_{0}\right)
$$

(recall that when $a_{2} a_{4} \neq 0, \alpha_{L}^{2}=\alpha_{R}^{2} \equiv \alpha^{2}$ ). From the $T_{6}$ gauging we obtain, as before, the constraints $d x_{0}=0$. As to the other generator, the corresponding current components are $\left(\right.$ for $\left.d x_{0}=0\right)$

$$
\begin{aligned}
J_{m}^{+}=\left(J_{+}^{L}, \alpha_{R} T_{m}\right)= & \alpha_{R}\left[\left(a_{4} e^{-x_{0}}-\frac{1}{2} a_{2} e^{x_{0}} x_{1}^{2}\right) \partial_{+} x_{2}\right. \\
& \left.+a_{2} e^{x_{0}}\left(-x_{1} \partial_{+} x_{3}+\partial_{+} x_{4}\right)\right] \\
J_{m}^{-}=\left(\alpha_{L} T_{m}, J_{-}^{R}\right)= & \alpha_{L}\left[-a_{2} x_{3} \partial_{-} x_{1}+a_{4} \partial_{-} x_{2}+a_{2} \partial_{-} x_{4}\right]
\end{aligned}
$$

and we have several different situations:

\footnotetext{
${ }^{40}$ More precisely, the basis that corresponds to the action $(4.31)$ is

$$
\left\{T_{a}^{L}\right\}=\left\{T_{m}, T_{6}, 0,0\right\} \quad, \quad\left\{T_{a}^{R}\right\}=\left\{0,0, T_{m}, T_{6}\right\},
$$

where the first two generators generate the left action and the other two generate the right action. The choice (4.33) corresponds to vectorial gauging of the central element: $\theta_{L}=\theta_{R}$ and to some left-right correlated gauging of $T_{m}: \varphi_{L, R}=\alpha_{L, R} \varphi$. The last restriction is actually necessary when $T_{m}$ is not null, while for null generators $\left(T_{0}\right.$ and possibly also $\left.T_{m}\right)$ we have seen that the resulting action is unaffected by these restrictions. Therefore, there is no loss of generality in the choice (4.33).
} 
1. $a_{2}=0$ :

This implies $M=0$, which leads to the constraints $J_{m}^{+}=J_{m}^{-}=0$. For $\alpha_{L} \alpha_{R} \neq 0$ this is equivalent to $d x_{2}=0$. Imposing these constraints in the action $\hat{S}$ eliminates the $x_{4}$ dependence and we obtain a 3 -dimensional $\sigma$-model action:

$$
\begin{aligned}
\hat{S}_{\text {eff }}\left(x_{1}, x_{3}, x_{5}\right) & =\left.S\right|_{d x_{0}=d x_{2}=0}= \\
& =\frac{\hbar}{4 \pi} \int_{\Sigma} d^{2} \sigma\left[2 \partial_{+} x_{5} \partial_{-} x_{1}+\partial_{+} x_{3} \partial_{-} x_{3}+2 x_{2} \partial_{+} x_{3} \partial_{-} x_{1}\right]
\end{aligned}
$$

which corresponds to a constant (flat) background:

$$
E_{\mu \nu}=\left(\begin{array}{ccc}
0 & x_{2} & 1 \\
x_{2} & 1 & 0 \\
1 & 0 & 0
\end{array}\right)
$$

2. $\alpha_{L} \alpha_{R}=0$ :

This requires $a_{2} a_{4}=0$ ( $T_{m}$ null $)$ and, therefore, also in this case $M$ vanishes. But in the present case either $T_{m}^{L}$ or $T_{m}^{R}$ vanish, therefore, we obtain only one constraint:

$$
\begin{array}{ll}
a_{2}=0: & \alpha_{L} \partial_{-} x_{2}=\alpha_{R} \partial_{+} x_{2}=0 \\
a_{4}=0: & \alpha_{L}\left(\partial_{-} x_{4}-x_{3} \partial_{-} x_{1}\right)= \\
& =\alpha_{R}\left(\partial_{+} x_{4}-x_{1} \partial_{+} x_{3}-\frac{1}{2} x_{1}^{2} \partial_{+} x_{2}\right)=0
\end{array}
$$

which eliminates roughly "half" a degree of freedom, therefore, it seems that the resulting model is not of a $\sigma$-model type.

3. $a_{2}, \alpha_{L} \alpha_{R} \neq 0$ :

(note that this includes the null case $a_{4}=0$ )

In this case $M$ does not vanish identically and the effect of the gauging (after integrating out the gauge field) is to add to the WZNW Lagrangian the contribution

$$
\frac{\hbar}{2 \pi}\left[\frac{1}{M} J_{m}^{+} J_{m}^{-}+(\log M) \partial_{+} \partial_{-} \varphi\right]
$$

\subsubsection{A Four Dimensional Model}

We continue with the last case. To choose an appropriate gauge fixing condition, we need the explicit action of $H$ in $G$. Using repeatedly a generalization of eq. (4.6):

$$
e^{S} f(T) e^{-S}=f\left(e^{\operatorname{ad}(S)}(T)\right)
$$

(valid for any function $f$ expressible as a convergent power series) and the CampbellBaker-Hausdorff formula [25], which for $[S, T]$ that commutes with $S$ and $T$ takes the form

$$
e^{S} e^{T}=s^{S+T+\frac{1}{2}[S, T]},
$$


we obtain

$$
\begin{aligned}
& x_{0} \rightarrow x_{0} \\
& x_{1} \rightarrow x_{1} \\
& x_{2} \rightarrow x_{2}+a_{2}\left(\varphi_{L}-e^{x_{0}} \varphi_{R}\right) \\
& x_{3} \rightarrow x_{3}+a_{2} e^{x_{0}} x_{1} \varphi_{R} \\
& x_{4} \rightarrow x_{4}+a_{4}\left(\varphi_{L}-e^{-x_{0}} \varphi_{R}\right)+\frac{1}{2} a_{2} e^{x_{0}} x_{1}^{2} \varphi_{R} \\
& x_{5} \rightarrow x_{5}-a_{2}\left(x_{3} \varphi_{L}+e^{x_{0}} x_{1} x_{2} \varphi_{R}\right)+a_{2}^{2} e^{x_{0}} x_{1}\left(\frac{1}{2} e^{x_{0}} \varphi_{R}-\varphi_{L}\right) \varphi_{R} \\
& x_{6} \rightarrow x_{6}+\left(\theta_{L}-\theta_{R}\right)+a_{2} x_{4} \varphi_{L}+\left(\frac{1}{2} a_{2} e^{x_{0}} x_{1}^{2}-a_{4} e^{-x_{0}}\right) x_{2} \varphi_{R} \\
&+\frac{1}{2} a_{2}^{2} e^{x_{0}} x_{1}^{2}\left(\varphi_{L}-\frac{1}{2} e^{x_{0}} \varphi_{R}\right) \varphi_{R}+a_{2} a_{4}\left(\varphi^{2}-e^{-x_{0}} \varphi_{L} \varphi_{R}\right) .
\end{aligned}
$$

Since we chose $a_{2} \neq 0$, one may fix $x_{2}$ as a gauge condition, unless $e^{x_{0}}=\alpha_{L} / \alpha_{R}$. At this stage we should observe that $x_{0}$ is not a fixed parameter of the theory but rather an integration variable - it is the remnant of the $\left[d x_{0}\right]$ functional integration (in the partition function), which was not fixed by the constraint $d x_{0}=0$. A single isolated value of $x_{0}$ has measure zero and does not have any influence on the integral. Therefore, we may restrict ourselves to the generic case

$$
e^{x_{0}} \neq \alpha_{L} / \alpha_{R}
$$

We choose the gauge $x_{2}=$ const., impose the constraint $x_{0}=$ const., and obtain the $\sigma$ model action

$$
\begin{aligned}
\hat{S}_{\mathrm{eff}}\left(x_{1}, x_{3}, x_{4}, x_{5}\right)= & \frac{\hbar}{4 \pi} \int_{\Sigma} d^{2} \sigma\left[2 \partial_{+} x_{5} \partial_{-} x_{1}+\partial_{+} x_{3} \partial_{-} x_{3}+2 x_{2} \partial_{+} x_{3} \partial_{-} x_{1}\right. \\
& \left.+4 p\left(x_{1} \partial_{+} x_{3}-\partial_{+} x_{4}\right)\left(x_{3} \partial_{-} x_{1}-\partial_{-} x_{4}\right)+2 \log \left(x_{1}^{2}+a\right) \partial_{+} \partial_{-} \varphi\right]
\end{aligned}
$$

with

$$
\begin{gathered}
p=\frac{\alpha_{L} \alpha_{R} a_{2}^{2}}{2 M} e^{x_{0}}=\frac{1}{x_{1}^{2}+a} \\
a=4 \frac{a_{4}}{a_{2}} e^{-x_{0}}\left(\frac{\alpha_{L}}{\alpha_{R}}-\cosh x_{0}\right)=-2 \frac{a_{4}}{a_{2}}\left(\frac{\alpha_{L}}{\alpha_{R}}-e^{-x_{0}}\right)^{2}
\end{gathered}
$$

(we used the fact that when $a_{4} \neq 0$, the anomaly condition imposes the constraint $\left.\alpha_{L} / \alpha_{R}= \pm 1\right)$. Note that all the parameters that determine the $T_{m}$-gauge are concentrated in one parameter $a$. Its sign is equal to the sign of $-\left(T_{m}, T_{m}\right)$ (since the other factors are strictly positive by assumption). We now show that the magnitude of $a$ carries no physical information, therefore, only its sign is important. Indeed, the action (4.47) is invariant under the transformation 4 ]

$$
x_{i} \rightarrow \lambda^{\hat{i}} x_{i}, a \rightarrow \lambda^{2} a \quad \lambda \neq 0
$$

${ }^{41}$ This transformation corresponds to a change of basis $T_{i} \rightarrow \lambda^{-\hat{i}} T_{i}$ in $\mathcal{A}_{n}$. For $\lambda>0$ this is the adjoint action of $\lambda^{T_{0}} \in G_{n}$ in $\mathcal{A}_{n}$, which is always an (inner) isometric automorphism (i.e. conserves the metric and the Lie bracket). For $\lambda=-1$ a simple check shows that it is also an isometric automorphism (although outer). This explains the invariance of the action (although it can be also easily verified directly). 
(where $\hat{i} \equiv i \bmod 3 \in\{-1,0,1\}$ ), therefore, a change in the magnitude of $a$ is equivalent to a coordinate transformation. Furthermore, for positive $\lambda$ the coordinate transformation (this time keeping $a$ unchanged!) is a result of the adjoint action of $\lambda^{-T_{0}}$ in $G_{n}$

$$
g \rightarrow \lambda^{T_{0}} g \lambda^{-T_{0}} .
$$

The functional measure $[d g]$ is invariant under such a transformation, therefore, the partition function is independent of the magnitude of $a$. This has several important implications:

1. The action is essentially (as an integrand) independent of the value of $x_{0}$ and the $d x_{0}$ integration in the partition function is trivial ${ }^{2}$. Therefore, we can view $x_{0}$ as a fixed parameter in the action (void of any physical content) and not as an integration variable and the resulting effective action is indeed of the $\sigma$-model type.

2. The model is independent of the choice of $\alpha_{L}$ and $\alpha_{R}$ (as long as they don't vanish). In other words, we again have trivial vectorial/axial duality (and its generalization in the null case).

3. The model is almost independent of the choice of $a_{2}$ and $a_{4}$, which determine the direction of $T_{m}$ in the $\left(T_{2}, T_{4}\right)$ plane. Only the sign of $-a_{2} a_{4}$ (which is the signature of $\left.T_{m}\right)$ is significant.

The fact that $x_{0}$ can be treated as a parameter implies that the model (4.47) coincides with the WZNW model based on the 5-dimensional algebra (4.30) gauged by $\operatorname{sp}\left\{T_{m}\right\}$. This model was derived in [12], using a different basis for the algebra and a different parametrization of the group manifold. The action obtained using those choices belongs to a family of exactly conformal $\sigma$-model actions of the form

$$
S\left[u, v, y_{i}\right]=\frac{\hbar}{4 \pi} \int_{\Sigma} d^{2} \sigma\left\{k\left[2 \partial_{+} v \partial_{-} u+U_{i j}(u) \partial_{+} y_{i} \partial_{-} y_{j}\right]-4 \Phi(u) \partial_{+} \partial_{-} \varphi\right\},
$$

considered in [26]. Moreover, the action corresponding to the axial gauging was obtained in [26] as a special limit of the $\left[E_{2}^{c} \otimes U(1)\right] / U(1)$ (vectorially) gauged WZNW model. It was also shown in [12 that the actions obtained are related to 4-dimensional flat actions by duality [24].

Incidentally, observe that in the limit $a_{2} / a_{4} \rightarrow 0, p \rightarrow 0$ and we obtain the action (4.39) of the $a_{2}=0$ case. In both cases we have $x_{2}=$ const., however, for $a_{2} \neq 0$ this is a gauge choice and the model is explicitly independent of the value chosen for $x_{2}$. On the other hand, for $a_{2}=0, x_{2}$ is constrained to be a constant and one still must apriori integrate over its value. However, the fact that the $a_{2}=0$ model can be obtained as a limit of the $a_{2} \neq 0$ model implies that the $a_{2}=0$ model is also independent of $x_{2}$ and the integration is unnecessary. In the present model this is trivially seen directly, but in

\footnotetext{
${ }^{42}$ We did not consider the value $e^{x_{0}}=\alpha_{L} / \alpha_{R}$ but, as explained already, this is a single point in the $d x_{0}$ integral and, therefore, can not have any influence.
} 
more complicated models, where the independence may not be obvious, this may be a convenient way to prove it.

For $a=0\left(a_{4}=0\right)$ the metric of the model (4.47) is degenerate, but as in the $\mathcal{A}_{3}$ models, this is because in this case $T_{m}$ is null (proportional to $T_{2}$ ), and we have an extended gauge symmetry $\left(\varphi_{L}, \varphi_{R}\right.$ independent in (4.31)). According to (4.45) (with $a_{4}=0$ ), for $x_{1} \neq 0$ one can choose the gauge $x_{2}=x_{3}=0$ and the resulting action is

$$
\hat{S}_{\mathrm{eff}}\left(x_{1}, x_{4}, x_{5}\right)=\frac{\hbar}{4 \pi} \int_{\Sigma} d^{2} \sigma\left[2 \partial_{+} x_{5} \partial_{-} x_{1}+\frac{4}{x_{1}^{2}} \partial_{+} x_{4} \partial_{-} x_{4}+4 \log \left(x_{1}\right) \partial_{+} \partial_{-} \varphi\right] .
$$

\subsubsection{Non-Abelian gauging}

In the search for a $\sigma$-model with a physical signature, one may consider also non-Abelian subalgebras. However, since $\mathcal{A}_{n}$ is solvable, its two and three dimensional subalgebras are not self-dual and, therefore, one expects a contribution from the trace anomaly [23]. We considered two examples, $d H=\operatorname{sp}\left\{T_{0}, T_{2}\right\}$ and $d H=\operatorname{sp}\left\{T_{0}, T_{5}\right\}$. Both of them are of the type $[T, S]=S$. The trace of (the adjoint representation of) $T$ is non-trivial, so the trace anomaly contributes and the $\sigma$-model background fields calculated according to the formulas in section 3 are expected not to satisfy the beta function equations. This is indeed what happens for the first model $\left(d H=\operatorname{sp}\left\{T_{0}, T_{2}\right\}\right)$. The second model leads to a constant (flat) $E_{\mu \nu}$ and a linear dilaton. The one-loop beta functions for such a background vanish with a shift in the central charge (relative to the dimension of the $\sigma$ model target manifold). We expect the trace anomaly contribution to cancel the dilaton, leading to a flat background.

\section{Summary and Remarks}

In this work we investigated WZNW and gauged WZNW models based on non-reductive algebras. We introduced a family $\left\{\mathcal{A}_{3 m}\right\}$ of such algebras that are not double extensions of Abelian algebras and, therefore, cannot be obtained through a Wigner contraction. This may provide one with a new family of conformal field theories.

We constructed WZNW and gauged WZNW models based on the first two algebras in this series: $\mathcal{A}_{3}$ and $\mathcal{A}_{6}$. The purpose was to find models that can serve as string vacua, and also to gain general knowledge about the use of non-reductive algebras (and the family $\mathcal{A}_{n}$ in particular) in this context. This indeed provided some general observations, which lead to the derivation of some general results concerning singular and central gauging. Here we describe some of the features and problems of the use of non-reductive algebras for the construction of WZNW and gauged WZNW models:

- The $\sigma$-model obtained from a WZNW model based on a non-reductive algebra is never positive definite. Moreover, in the process of constructing a non-reductive self-dual algebra, starting from an Abelian one, each double extension adds at least one timelike direction. This implies that (indecomposable) non-reductive algebras, 
that are not double extensions of Abelian algebras, always lead to an unphysical signature, with more than one time-like direction, so to obtain a useful model using these algebras, one must gauge out the extra time-like directions (see, for example [7]).

- Many of the possibilities of gauging a non-reductive WZNW model are singular. That singular subalgebras are quite common can be seen in the $\mathcal{A}_{n}$ algebras and is also suggested by the structure of a double extension. In fact, lower dimensional self dual subalgebras are quite rare, as can be seen in the list of [12]: two dimensional non-Abelian sub-algebras are never self-dual and if the (total) algebra is solvable (and, therefore, does not contain simple subalgebras), this is true also for three dimensional subalgebras.

- When the gauging is singular the gauge symmetry group of the model is typically larger then the subgroup initially chosen to be gauged and as a result such a gauging reduces the dimension of the $\sigma$-model target manifold by more than the dimension of that subgroup. For example, when $H_{L}$ and $H_{R}$ are one dimensional and null, The dimension is reduced by two.

- The signature of a $\sigma$-model obtained from a gauged WZNW seems to be related to the one obtained from the ungauged model in a simple way:

- when the gauging is non-singular (i.e. , the metric induced on the algebra of the gauged subgroup is non-degenerate), the signature of the gauged model is obtained from the ungauged one by "subtracting" the signature of the gauged subalgebra;

- when the gauging is null (and leads to a $\sigma$-model) the dimension of the target manifold reduces in pairs, consisting of one positive and one negative direction.

Therefore, the choice of a gauged subgroup is restricted to subgroups with the signature dictated by the desired final signature. In particular, this limits the use of null directions. In the $\mathcal{A}_{n}$ algebras, for example, the difference between the number of positive and negative eigenvalues of the metric is 0 or 1 , and to get a 4-dimensional (or larger) Minkowskian-signature background, one must gauge a non-null sub-algebra.

- Singular gauging leads frequently (although not always) to the appearance of constraints which, in many cases, lead to a model that is not of a $\sigma$-model type. All possible situations were demonstrated in the $\mathcal{A}_{6}$ models described in section 4 :

$-d H_{L}=\operatorname{sp}\left\{T_{4}\right\}, d H_{R}=0$ (or vise versa) leads to constraints that lead to a non- $\sigma$-model system;

- $d H_{L}=d H_{R}=\operatorname{sp}\left\{T_{4}\right\}$ leads also to constraints but these $d o$ lead to a $\sigma$-model;

$-d H_{L}=d H_{R}=\operatorname{sp}\left\{T_{2}\right\}$ does not lead to constraints. 
Another complication is that in singular gaugings, the gauged subgroup is not necessarily self-dual and when it is not, there may be a non-trivial contribution from the trace anomaly [23] (as demonstrated at the end of section 4). However, there are singularly gauged WZNW models that $d o$ lead to a good $\sigma$-model, so this possibility does not have to be completely avoided.

- Gauging a central subgroup does not lead to new $\sigma$-models, at least for $H_{L}=H_{R}$. It either leads to an ungauged WZNW model or does not lead to a clearly defined field theory at all. For example, gauging the $T_{6}$ direction of $\mathcal{A}_{6}$ lead to the WZNW model for $\mathcal{A}=\operatorname{sp}\left\{T_{1}, \ldots, T_{6}\right\} / \operatorname{sp}\left\{T_{6}\right\}$. We also gauged a subalgebra of $\mathcal{A}_{6}$ that contained the central element $T_{6}$, and the result coincided with a gauged WZNW model based on $\mathcal{A}$. This seems as a general result. Therefore, to obtain genuine new models, it seems that one should avoid subgroups containing central elements. If this is true, it limits considerably the useful choices of gauging.

In spite of the problems and limitations encountered, gauged WZNW models based on the algebras $\left\{\mathcal{A}_{n}\right\}$ and on non-reductive self-dual algebras in general, may lead to new and interesting string backgrounds (as was already demonstrated) and therefore deserve further study. In particular, one may try to derive string backgrounds using the next algebras of the family $\mathcal{A}_{n}$. These are not double extensions of Abelian algebras and it would be interesting to see if this property is reflected in some way in the WZNW models (or in other models based on non-reductive self-dual algebras [13).

Finally, we should remark that an open direction for research is the construction of the conformal field theory that corresponds to the gauged WZNW model. For non-singular vectorial gauging this is the coset construction (for a non-reductive self-dual algebra, this was shown in [14]), but for the other gauging possibilities the corresponding CFT is not known. In particular it is not clear what is the resulting central charge. For non-singular vectorial gauging it is equal to the difference between the central charges of WZNW models based on the group $G$ and on the subgroup $H$ respectively. When $G$ is solvable, this implies that the central charge is equal to the dimension of the $\sigma$-model target manifold. All the $\sigma$-models derived in section 4 obeyed this rule. It remains to be seen if this is true in general.

\section{Acknowledgment}

We would like to thank A.A. Tseytlin for useful discussions. The work of AG is supported in part by BSF - American-Israel Bi-National Science Foundation, by the BRF - the Basic Research Foundation and by an Alon fellowship. The work of ER is supported in part by BSF and by the BRF. 


\section{Appendix A. Comments and Supplements}

\section{A.1 Generalizations of the algebras $\mathcal{A}_{n}$}

Here we comment about possible generalizations of the algebras defined in section 2.2, obtained by using the defining relations (2.23) with a different choice of the map "^". If one takes "^" to be some homomorphism from $\mathbb{Z}$ to some commutative ring $\mathbb{F}$ with unity, (2.24-2.26) hold, as well as (2.29) and one obtains a Lie algebra over $\mathbb{F}$. For example, one can take $\mathbb{F}=\mathbb{Z}_{p}$ ( $p$ a positive integer) with "^" being the natural homomorphism. This example, however, is irrelevant for WZNW models, since one needs there an algebra over $\mathbb{R}$ and for this $\mathbb{F}$ must be some subring of $\mathbb{R}$. A more relevant example will be obtained by taking $\mathbb{F}=\mathbb{Z}$ and "^" the identity map. The result is the Virasoro algebra (with zero central charge). Another natural candidate for "^" would be $\hat{i}=i \bmod p$ ( $p$ a positive integer). Taking $p=2$ and ${ }^{\wedge}: \mathbb{Z} \rightarrow\{0,1\}$, an analysis similar to the $p=3$ case leads to the choice $(i, j, k)=(1,0,0)$, for which the right-hand-side of (2.29) does not vanish $\left(\hat{c}_{i j k}=\hat{c}_{k i j}=1, \hat{c}_{j k i}=0\right)$. There seems to be no other choice of $p$ and range of the map "^" such that the multiplication is preserved. In the main text we concentrate on the specific choice $\hat{i}=i \bmod 3 \in\{-1,0,1\}$, but we rarely use more then the properties $(2.24 \sqrt{2.26})$ and the Jacobi identity (2.29), so most of the analysis applies to possible future generalizations.

\section{A.2 A WZNW model with a degenerate metric}

Here we analyze the WZNW model (section 3.1) obtained when one uses a degenerate metric. Let us define

$$
\mathcal{J} \equiv\{R \in d G \mid(R, T)=0, \forall T \in d G\} .
$$

The invariance of the metric implies that this is an ideal, therefore, it corresponds to a normal subgroup $N$ of $G: \mathcal{J}=d N$; and the metric $(\cdot, \cdot)$ is an invertible metric on the algebra $d G_{0}$ of the quotient group $G_{0} \equiv G / N$. Taking for an element in $G$ a parametrization of the form $g=n g_{0}$, where $n \in N$ and $g_{0}$ parametrizes elements of the quotient group, it is straightforward to show that the action is independent of $n$, therefore, it actually corresponds to the group $G_{0}$ (for which the metric is non-degenerate). Therefore, relaxing the requirement of non-degeneracy gives us nothing new.

\section{A.3 Conditions for the appearance of constraints}

Here we show that for a wide class of gauged WZNW models, constraints do not appear and, therefore, the resulting model is a non linear $\sigma$-model. We find that when an appropriate single "diagonalizing" basis $T_{a}^{+}=T_{a}^{-}$for $d H$ exists (and in particular for axial and vector gauging), constraints can appear only if $G$ is not semi-simple or the gauging is singular (i.e. the metric $(\cdot, \cdot)_{d H}$ induced on $d H$ is degenerate). As we saw in section 3.2, 
the appearance of constraints is equivalent to the degeneracy of the matrix $M_{a b}$, which means that there exist $T_{\breve{a}}^{ \pm} \in d H$ for which

$$
T_{\check{a}}^{L-}-g T_{\check{a}}^{R-} g^{-1} \perp d H_{L} \quad, \quad T_{\check{a}}^{R+}-g^{-1} T_{\breve{a}}^{L+} g \perp d H_{R} .
$$

Assuming $T_{\breve{a}}^{+}=T_{\breve{a}}^{-}$, this implies

$$
T_{\check{a}}^{L}-T_{\check{a}}^{R} \perp d H_{L}+d H_{R}
$$

and when $(\cdot, \cdot)_{d H}$ is non-degenerate, this implies $T_{\breve{a}}^{L}=T_{\breve{a}}^{R}$. Putting this in (A.1), we obtain

$$
g T_{\check{a}} g^{-1}-T_{\check{a}} \perp d H_{L}+d H_{R}
$$

which means that the space

$$
\mathcal{J} \equiv \operatorname{sp}\left\{g T_{\check{a}} g^{-1}-T_{\check{a}}\right\}_{g \in G}
$$

is orthogonal to $d H_{L}+d H_{R}$. Using relations (4.5) and (4.6), we obtain, for any $S \in d G$

$$
\left[S, g T_{\check{a}} g^{-1}-T_{\check{a}}\right]=\lim _{t \rightarrow 0} \frac{1}{t}\left\{\left[\left(e^{t S} g\right) T_{\check{a}}\left(e^{t S} g\right)^{-1}-T_{\check{a}}\right]-\left[e^{t S} T_{\check{a}} e^{-t S}-T_{\check{a}}\right]-\left[g T_{\check{a}} g^{-1}-T_{\breve{a}}\right]\right\}
$$

which means that $\mathcal{J}$ is an ideal in $d G$. If $G$ is semi-simple, $T_{\breve{a}}$ is not central, therefore, $\mathcal{J}$ is not empty (since it contains $\left[d G, T_{\check{a}}\right]$ ), so $\mathcal{J}$ is a semi-simple factor:

$$
d G=\mathcal{J} \oplus \mathcal{J}^{\prime} \quad, \quad \mathcal{J} \perp \mathcal{J}^{\prime}
$$

and $d H_{L}+d H_{R}$ is contained in the other factor $\mathcal{J}^{\prime}$. This implies that $T_{\breve{a}}$ is in $\mathcal{J}^{\prime}$ and, therefore, so is $g T_{\breve{a}} g^{-1}$ (since $\mathcal{J}$ is an ideal). This would imply that $\mathcal{J}$ is contained in $\mathcal{J}^{\prime}$, in contradiction to (A.6) and the non-triviality of $\mathcal{J}$. Thus in this case $T_{\check{a}}$ as above cannot exist.

\section{A.4 Vectorial gauging of a central group}

When the center $Z$ of $G$

$$
Z=\{c \in G \mid c g=g c \forall g \in G\}
$$

is non-trivial, then $Z$, embedded diagonally in $G_{L} \otimes G_{R}$, acts trivially in $G$ :

$$
h g h^{-1}=g \forall h \in Z, g \in G
$$

and the faithfully acting symmetry group of the action (3.1) is $G_{L} \otimes G_{R} / Z$. Therefore, it seems meaningless to "vectorially gauge" the center (or a subgroup of it) since the original action is already (trivially) gauge invariant. In spite of this, the gauged action (3.7) is different from the ungauged one 43 :

$$
\hat{S}[g, A]=S[g]+\frac{\hbar}{2 \pi} \int_{\Sigma} d^{2} \sigma \epsilon^{\alpha \beta}\left(A_{\alpha}, J_{\beta}^{L}\right) .
$$

\footnotetext{
${ }^{43}$ The difference should be separately gauge invariant, and indeed it is - as can be directly verified.
} 
Moreover, we showed in section 3.3 that when the gauged group is null, "vectorial" gauging is identical to "axial" gauging, where the $g$ and $S$ are not gauge invariant. The question that arises is, therefore, what does it mean, in this context, to gauge vectorially a central group.

The situation becomes more clear when we recall that the purpose of the introduction of the gauge fields in a WZNW model is not to obtain a gauge invariant theory but rather to obtain a model with a reduced dimension. The simplest example is that of "axial", non-singular gauging of a central group $C$. We have shown in section 3.4 that in such a situation $d G$ is an orthogonal direct sum of ideals $d G_{0} \oplus d C$ and, therefore, $G$ has a parametrization $g=g_{0}(x) e^{z^{i} T_{i}}$ where $g_{0}(x)$ is some parametrization of $G_{0}$ and $\left\{T_{i}\right\}$ is a basis for $d C$. The symmetry gauged is

$$
g \rightarrow e^{2 \theta^{i} T_{i}} g(=h h g=h g h)
$$

which is equivalent to $z^{i} \rightarrow z^{i}+2 \theta^{i}$. After fixing the gauge (and integrating out the gauge fields) we are left with a WZNW model for $G_{0}$.

In the vectorial case the situation is quite different but the final result is identical: keeping in mind the real purpose of the gauging procedure, we add the additional terms to the (already gauge-invariant) ungauged action. Since $g$ is gauge-invariant, the dimension can not be reduced by gauge fixing. Instead, the elimination of physical degrees of freedom occurs here because of the appearance of constraints, introduced by the additional terms. Indeed, we have

$$
\hat{S}[g, A]=S[g]+\frac{\hbar}{2 \pi} \int_{\Sigma}\left(A, T_{i}\right) \wedge d z^{i}
$$

therefore, the integration of the gauge fields leads to the constraints $d z^{i}=0$, which results, again, with a WZNW model for $G_{0}$. To summarize, we have shown that "vectorial gauging" of a central group has a well defined meaning in the present context, although the name "gauging" is misleading. We also saw that in all cases of central gauging (singular or not), vector/axial duality is not only valid, but also trivial.

\section{A.5 Gauging a one dimensional central group with $H_{L} \neq H_{R}$}

In section 3.4 we analyzed central gauging when $H_{L}=H_{R}$. To get an idea what new behavior can be expected, when $H_{L}$ and $H_{R}$ are different, we examine here the one dimensional case.

We define (as in (3.38) ) the group

$$
\bar{H}=H_{L} H_{R} \equiv\left\{h_{L} h_{R} \mid h_{L, R} \in H_{L, R}\right\}
$$

(which is also a central subgroup of $G$ ) and analyze different situations according to the rank of the metric on

$$
d \bar{H}=\operatorname{sp}\left\{T_{L}, T_{R}\right\}
$$


- $\bar{H}$ is null $\left((\cdot, \cdot)_{d \bar{H}}=0\right)$

This case was analyzed (for arbitrary $\operatorname{dim} \bar{H}$ ) in a footnote after eq. (3.37). It contains the case of "one sided gauging" (when one of the groups $H_{L}$ or $H_{R}$ vanishes). The effect of the gauging is that some of the coordinates will be constrained to depend on one of the light-cone coordinates and it is not clear if the resulting action can be brought to the form of a $\sigma$-model.

- $(\cdot, \cdot)_{d \bar{H}}$ is non-degenerate

This implies that

$$
d G=d G_{0} \oplus d \bar{H} \quad, \quad d G_{0} \perp d \bar{H},
$$

which is of the form (3.31) and the result is:

- when $H$ is null: a WZNW model for $G_{0}$.

- when $H$ is not null:

a WZNW model for $G_{0}$, tensored with a one dimensional free model.

- $\operatorname{rank}(\cdot, \cdot)_{d \bar{H}}=1$

This is the only case essentially different from the $H_{L}=H_{R}$ ones. We choose a diagonalizing basis for $d \bar{H}$

$$
d \bar{H}=\operatorname{sp}\left\{T_{0}, T_{1}\right\} \quad, \quad\left(T_{i}, T_{j}\right)=i \delta_{i j}
$$

(note that because of the anomaly condition, $\bar{H}$ is necessarily two dimensional in this case) and obtain the structure (3.30) with $H_{i}=\operatorname{sp}\left\{T_{i}\right\}$. The anomaly condition implies

$$
T_{L}=\alpha T_{1}+\beta_{L} T_{0} \quad, \quad T_{R}= \pm\left(\alpha T_{1}+\beta_{R} T_{0}\right)
$$

and $H_{L} \neq H_{R}$ implies $\beta_{L} \neq \beta_{R}$ and $\alpha \neq 0$. We observe that $T_{0}^{\perp}$ (the subspace of $d G_{0}$ orthogonal to $T_{0}$ ) is a subalgebra containing $T_{0}$, therefore, corresponds to a subgroup of $G_{0}$, which we denote by $K$. Next we choose some element $S$ of $d G_{0}$ obeying $\left(S, T_{0}\right)=1$ and obtain the structure

$$
d G_{0}=\operatorname{sp}\{S\} \in d K
$$

which suggests the following parametrization for $g \in G$

$$
g\left(x, y, z_{0}, z_{1}\right)=k(x) e^{y S+Z_{i} T_{i}},
$$

where $\mathrm{k}(\mathrm{x})$ is some parametrization of the quotient group $K / \operatorname{sp}\left\{T_{0}\right\}$. In this parametrization, the invariant form $J^{L}$ is

$$
J^{L}=T_{i} d z_{i}+S d y+J_{\perp}^{L} \quad, \quad J_{\perp}^{L}(x, y)=e^{-y S} k^{-1} d k e^{y S} \in d K
$$

and this leads, in the notation of section 3.2 to

$$
M=\alpha^{2}(1 \mp 1) \quad, \quad J^{+}= \pm \partial_{+}\left(\alpha z_{1}+\beta_{L} y\right) \quad, \quad J^{-}=\partial_{-}\left(\alpha z_{1}+\beta_{R} y\right) .
$$


The coordinate transformation representing the action of $H$

$$
g \rightarrow e^{\theta T_{L}} g e^{-\theta T_{R}}
$$

is

$$
\delta z_{0}=\theta\left(\beta_{L} \mp \beta_{R}\right) \quad, \quad \delta z_{1}=\theta \alpha(1 \mp 1) .
$$

For the vector-like gauging (the upper sign), $M=0$ and one obtains (gaugeinvariant) constraints $J^{+}=J^{-}=0$ that seem not to lead to a $\sigma$-model. For the axial-like gauging (the lower sign), $M \neq 0$ and the effective action is

$$
\hat{S}_{\mathrm{eff}}=S[g]+\frac{\hbar}{2 \pi} \int_{\Sigma} d^{2} \sigma \frac{J^{+} J^{-}}{M} .
$$

With the gauge choice $z_{1}=0, S[g]$ becomes the WZNW action for $G_{0}$ and the effect of the second term is to change the $\sigma$-model metric

$$
d s^{2} \rightarrow d s^{2}-\frac{\beta_{L} \beta_{R}}{\alpha^{2}} d y^{2},
$$

which is equivalent to a change in the value of the norm (S,S). This value can be changed by the automorphic redefinition $S \rightarrow S+\gamma T_{0}$, therefore, the change does not effect the invariance and non-degeneracy of the metric. We conclude that the resulting model is a WZNW model for $G_{0}$ with a modified invariant metric.

To summarize, we found that in all the cases of one dimensional central gauging, one either encounters constraints that seem not to lead to a $\sigma$-model, or obtains an ungauged WZNW model.

\section{Appendix B. The $\sigma$ model backgrounds obtained}

In section 4 we derived several models of the $\sigma$-model type:

$$
\begin{gathered}
S[x]=\frac{\hbar}{8 \pi} \int_{\Sigma} d^{2} \sigma\left[\left(\sqrt{|h|} h^{\alpha \beta} G_{\mu \nu}+\epsilon^{\alpha \beta} B_{\mu \nu}(x)\right) \partial_{\alpha} x^{\mu} \partial_{\beta} x^{\nu}+\sqrt{|h|} R^{(2)} \Phi(x)\right] \\
=\frac{\hbar}{4 \pi} \int_{\Sigma} d^{2} \sigma\left[E_{\mu \nu}(x) \partial_{+} x^{\mu} \partial_{-} x^{\nu}-4 \Phi(x) \partial_{+} \partial_{-} \varphi\right], \\
E_{\mu \nu}=G_{\mu \nu}+B_{\mu \nu} .
\end{gathered}
$$

In this Appendix we list, for each model, the corresponding background fields: the metric $G_{\mu \nu}$, the anti-symmetric tensor $B_{\mu \nu}$ and the dilaton $\Phi$, and some related quantities (indexes are lowered by $G_{\mu \nu}$ and raised by the inverse metric $G^{\mu \nu}$ ):

- the connection

$$
\Gamma_{\mu \nu}^{\rho}=\frac{1}{2} G^{\rho \sigma}\left(\partial_{\mu} G_{\nu \sigma}+\partial_{\nu} G_{\mu \sigma}-\partial_{\sigma} G_{\mu \nu}\right) ;
$$


- the Riemann tensor

$$
R_{\mu \nu \rho}^{\sigma}=2\left(\Gamma_{\rho[\mu}^{\lambda} \Gamma_{\nu] \lambda}^{\sigma}-\partial_{[\mu} \Gamma_{\nu] \rho}^{\sigma}\right) ;
$$

- the Ricci tensor $R_{\mu \nu}=R_{\mu \rho \nu}{ }^{\rho}$ and the Ricci scalar $R=R_{\mu \nu} G^{\mu \nu}$;

- the torsion of the anti-symmetric tensor

$$
H=3 d B=3 \partial_{[\rho} B_{\mu \nu]} d x^{\rho} \wedge d x^{\mu} \wedge d x^{\nu}
$$

- contractions of the squared torsion

$$
H_{\mu \nu}^{2}=H_{\mu \rho \sigma} H_{\nu}^{\rho \sigma} \quad, \quad H^{2}=H_{\mu \nu}^{2} G^{\mu \nu} .
$$

These quantities are needed, among other things, to verify the one-loop beta function equations 20

$$
\begin{aligned}
0=\frac{16 \pi^{2}}{\alpha^{\prime}} \beta^{\Phi} & =\frac{c-d}{3 \alpha^{\prime}}+4(\nabla \Phi)^{2}-4 \nabla^{2} \Phi-R+\frac{1}{12} H^{2}+\mathcal{O}\left(\alpha^{\prime}\right) \\
0=\beta_{\mu \nu}^{G} & =R_{\mu \nu}-\frac{1}{4} H_{\mu \nu}^{2}+2 \nabla_{\mu} \nabla_{\nu} \Phi+\mathcal{O}\left(\alpha^{\prime}\right) \\
0=\beta_{\mu \nu}^{B} & =\nabla^{\rho} H_{\rho \mu \nu}-2\left(\nabla^{\rho} \Phi\right) H_{\rho \mu \nu}+\mathcal{O}\left(\alpha^{\prime}\right) .
\end{aligned}
$$

These equations were indeed found to be satisfied for all the models presented, with the central charge $c$ equal to the dimension $d$ of the target manifold.

\section{B.1 $\mathcal{A}_{3}$ ungauged}

(eq. (4.13))

The coordinates of the target manifold are

$$
\left\{x_{0}, x_{1}, x_{2}, x_{3}\right\}
$$

the background fields are:

$$
\begin{gathered}
G_{\mu \nu}=\left(\begin{array}{cccc}
b & 0 & x_{1} & 1 \\
0 & 0 & 1 & 0 \\
x_{1} & 1 & 0 & 0 \\
1 & 0 & 0 & 0
\end{array}\right) \\
\Phi=0
\end{gathered}
$$

( $B=2 x_{1} d x_{2} \wedge d x_{0}$; the metric with signature $(+,+,-,-)$, as the metric on $\left.\mathcal{A}_{3}\right)$; the inverse metric is

$$
G^{\mu \nu}=\left(\begin{array}{cccc}
0 & 0 & 0 & 1 \\
0 & 0 & 1 & -x_{1} \\
0 & 1 & 0 & 0 \\
1 & -x_{1} & 0 & -b
\end{array}\right)
$$


the only non-vanishing component of the Riemann tensor is (up to symmetry) $R_{0120}=\frac{1}{4}$, the only non-vanishing component of the Ricci tensor is $R_{00}=-\frac{1}{2}$ and the Ricci scalar vanishes.

The torsion of the anti-symmetric tensor is

$$
H=6 d x_{0} \wedge d x_{1} \wedge d x_{2}
$$

(which means $H_{012}=1$ ) and it is covariantly constant; the only non-vanishing component of $H_{\mu \nu}^{2}$ is $H_{00}^{2}=-2$ and $H^{2}$ vanishes.

\section{B.2 $\mathcal{A}_{3}$ gauged axially by $\operatorname{sp}\left\{T_{0}\right\}$}

(eq. (4.19))

The coordinates of the target manifold are

$$
\left\{x_{1}, x_{2}, x_{3}\right\}
$$

the background fields are:

$$
\begin{gathered}
G_{\mu \nu}=p\left(\begin{array}{ccc}
0 & 2 b & -x_{2} \\
2 b & 0 & -x_{1} \\
-x_{2} & -x_{1} & -2
\end{array}\right) \quad, \quad B_{\mu \nu}=p\left(\begin{array}{ccc}
0 & x_{1} x_{2} & x_{2} \\
-x_{1} x_{2} & 0 & -x_{1} \\
-x_{2} & x_{1} & 0
\end{array}\right) \\
\Phi=-\frac{1}{2} \log \left|x_{1} x_{2}+2 b\right|+\text { const. }
\end{gathered}
$$

where

$$
p=\frac{1}{x_{1} x_{2}+2 b}
$$

the metric has determinant $\operatorname{det} G_{\mu \nu}=4 b p^{2}$ and $\operatorname{signature}(+,-\operatorname{sign}(b),-)$, so the signature of the gauged model is obtained (when the gauging is non-singular) by "subtracting" the signature of $d H$ from that of $d G$; all background fields are singular at $x_{1} x_{2}=-2 b$. The inverse metric is

$$
G^{\mu \nu}=p\left(\begin{array}{ccc}
-\frac{x_{1}^{2}}{4 b} & 1+\frac{x_{1} x_{2}}{4 b} & -\frac{1}{2} x_{1} \\
1+\frac{x_{1} x_{2}}{4 b} & -\frac{x_{2}^{2}}{4 b} & -\frac{1}{2} x_{2} \\
-\frac{1}{2} x_{1} & -\frac{1}{2} x_{2} & -b
\end{array}\right)
$$

\footnotetext{
${ }^{44}$ The signature can be determined as follows. It can change only where the determinant vanishes or depends non-continuously on the coordinates. This happens only for $x_{1} x_{2}=-2 b$, so it is enough to check the signature for $x_{1}=x_{2}=0$ and for $x_{1} x_{2} / b \rightarrow \infty$. The first case is trivial. For the second case, we move to coordinates $(x, y, z)=\left(x_{3}, x_{1} x_{2}, x_{1} / x_{2}\right)$. The metric in these coordinates is

$$
\frac{p}{y}\left(\begin{array}{ccc}
-2 y & -y & 0 \\
-y & b & 0 \\
0 & 0 & -b\left(\frac{y}{z}\right)^{2}
\end{array}\right),
$$

so it has an eigenvalue with sign opposite to $b$. This rules out the signature $\operatorname{sign}(b)(+++)$, which is the only other possibility compatible with the sign of the determinant.
} 
the Ricci tensor is

$$
R_{\mu \nu}=p^{3}\left(\begin{array}{ccc}
0 & 8 b^{2} & x_{2}\left(x_{1} x_{2}-2 b\right) \\
8 b^{2} & 0 & x_{1}\left(x_{1} x_{2}-2 b\right) \\
x_{2}\left(x_{1} x_{2}-2 b\right) & x_{1}\left(x_{1} x_{2}-2 b\right) & 2\left(x_{1} x_{2}-2 b\right)
\end{array}\right)
$$

and the Ricci scalar is

$$
R=2 p^{2}\left(5 b-x_{1} x_{2}\right) .
$$

The torsion of the anti-symmetric tensor is $H_{123}=-4 b p^{2}$;

$$
H_{\mu \nu}^{2}=p^{3}\left(\begin{array}{ccc}
0 & 16 b^{2} & -8 b x_{2} \\
16 b^{2} & 0 & -8 b x_{1} \\
-8 b x_{2} & -8 b x_{1} & -16 b
\end{array}\right)
$$

and $H^{2}=24 b p^{2}$.

\section{B.3 $\mathcal{A}_{3}$ gauged vectorially by $\operatorname{sp}\left\{T_{0}\right\}$}

(eq. (4.21))

The coordinates of the target manifold are

$$
\left\{x_{0}, x_{2}, x_{3}\right\}
$$

the background fields are

$$
\begin{gathered}
G_{\mu \nu}=\frac{1}{x_{2}}\left(\begin{array}{ccc}
-b\left(x_{2}+2 b\right) & -b & -2 b \\
-b & 0 & -1 \\
-2 b & -1 & -2
\end{array}\right) \quad, \quad B_{\mu \nu}=\frac{1}{x_{2}}\left(\begin{array}{ccc}
0 & b & x_{2} \\
-b & 0 & -1 \\
-x_{2} & 1 & 0
\end{array}\right) \\
\Phi=-\frac{1}{2} \log \left|x_{2}\right|+\text { const.; }
\end{gathered}
$$

the metric has determinant $\operatorname{det} G_{\mu \nu}=4 b / x_{2}^{2}$ and $\operatorname{signature}(+,-\operatorname{sign}(b),-)$, as in the axial gauging;

the inverse metric is

$$
G^{\mu \nu}=\left(\begin{array}{ccc}
-\frac{1}{b} & 0 & 1 \\
0 & 2 x_{2} & -x_{2} \\
1 & -x_{2} & -b
\end{array}\right)
$$

the non-vanishing components of the Riemann tensor are (up to symmetry)

$$
R_{0202}=\frac{b^{2}}{x_{2}^{3}} \quad, \quad R_{0232}=\frac{b}{x_{2}^{3}} \quad, \quad R_{3232}=\frac{1}{x_{2}^{3}} ;
$$

\footnotetext{
${ }^{45}$ The other possibility $\operatorname{sign}(b)(+,+,+)$ is ruled out because, as in the axial case, the signature does not depend on $\left|x_{2}\right|$ and the sum of the eigenvalues is $\operatorname{tr} G_{\mu \nu}=-b+\mathcal{O}\left(1 / x_{2}\right)$, which for $\left|x_{2}\right|$ large enough has a sign opposite to $b$.
} 
the Ricci tensor is

$$
R_{\mu \nu}=\frac{1}{x_{2}^{2}}\left(\begin{array}{ccc}
2 b^{2} & b & 2 b \\
b & 0 & 1 \\
2 b & 1 & 2
\end{array}\right)
$$

and the Ricci scalar is $R=-\frac{2}{x_{2}}$.

The torsion of the anti-symmetric tensor vanishes.

\section{B.4 $\mathcal{A}_{3}$ gauged by null $\operatorname{sp}\left\{T_{0}\right\}$}

(eq. (4.22))

The coordinates of the target manifold are

$$
\left\{x_{2}, x_{3}\right\}
$$

the background fields are:

$$
\begin{gathered}
G_{\mu \nu}=-\frac{1}{x_{2}}\left(\begin{array}{cc}
0 & 1 \\
1 & 2
\end{array}\right) \quad, \quad B_{\mu \nu}=\frac{1}{x_{2}}\left(\begin{array}{cc}
0 & -1 \\
1 & 0
\end{array}\right) \\
\Phi=-\frac{1}{2} \log \left|x_{2}\right|+\text { const. }
\end{gathered}
$$

the metric has signature $(+,-)$, which means that this gauging eliminated one positive and one negative eigenvalue.

The inverse metric is

$$
G^{\mu \nu}=x_{2}\left(\begin{array}{cc}
2 & -1 \\
-1 & 0
\end{array}\right)
$$

the Riemann tensor is $R_{2323}=\frac{1}{x_{2}^{3}}$; the Ricci tensor is

$$
R_{\mu \nu}=\frac{1}{x_{2}^{2}}\left(\begin{array}{ll}
0 & 1 \\
1 & 2
\end{array}\right)
$$

and the Ricci scalar is $R=-\frac{2}{x_{2}}$.

The torsion of the anti-symmetric tensor vanishes.

\section{B.5 $\mathcal{A}_{6}$ ungauged}

(eq. (4.26))

The coordinates of the target manifold are

$$
\left\{x_{0}, x_{1}, x_{2}, x_{3}, x_{4}, x_{5}, x_{6}\right\}
$$


the background fields are

$$
\begin{aligned}
& G_{\mu \nu}=\left(\begin{array}{ccccccc}
b & 0 & 0 & x_{1} x_{2} & -x_{2} & x_{1} & 1 \\
0 & 0 & 0 & x_{2} & 0 & 1 & \\
0 & 0 & 0 & 0 & 1 & & \\
x_{1} x_{2} & x_{2} & 0 & 1 & & & \\
-x_{2} & 0 & 1 & & 0 & \\
x_{1} & 1 & & & 0 & \\
1 & & & & & &
\end{array}\right) . \\
& B_{\mu \nu}=\left(\begin{array}{ccccccc}
0 & 0 & 0 & -x_{1} x_{2} & x_{2} & -x_{1} & 0 \\
0 & 0 & 0 & 0 & 0 & 0 & \\
0 & 0 & 0 & x_{1} & 0 & & \\
x_{1} x_{2} & 0 & -x_{1} & 0 & & & \\
-x_{2} & 0 & 0 & & 0 & \\
x_{1} & 0 & & & & 0 \\
0 & & & & & &
\end{array}\right) \\
& \Phi=0 ;
\end{aligned}
$$

the inverse metric is

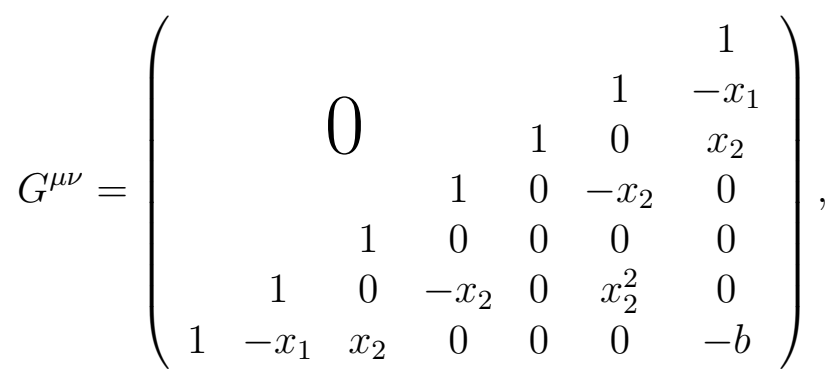

the non-vanishing components of the Riemann tensor are (up to symmetry)

$$
\begin{gathered}
R_{0103}=-\frac{x_{2}}{4}, \quad R_{0202}=\frac{x_{1}^{2}}{4}, \\
R_{0203}=R_{0212}=\frac{x_{1}}{4}, \\
R_{0150}=R_{0123}=R_{0240}=R_{0213}=R_{1212}=\frac{1}{4} ;
\end{gathered}
$$

the only non-vanishing component of the Ricci tensor is $R_{00}=-1$ and the Ricci scalar vanishes.

The non-vanishing components of the torsion are (up to symmetry)

$$
\begin{gathered}
H_{013}=x_{2} \quad, \quad H_{023}=x_{1}, \\
H_{015}=H_{042}=H_{123}=1
\end{gathered}
$$

and it is covariantly constant; the only non-vanishing component of $H_{\mu \nu}^{2}$ is $H_{00}^{2}=-4$ and $H^{2}$ vanishes. 


\section{B.6 $\mathcal{A}_{6}$ gauged by $\operatorname{sp}\left\{T_{6}\right\}$}

(eq. (4.29))

The coordinates of the target manifold are

$$
\left\{x_{1}, x_{2}, x_{3}, x_{4}, x_{5}\right\}
$$

the background fields are

$$
\begin{aligned}
G_{\mu \nu} & =\left(\begin{array}{ccccc}
0 & 0 & x_{2} & 0 & 1 \\
0 & 0 & 0 & 1 & \\
x_{2} & 0 & 1 & & \\
0 & 1 & & 0 & \\
1 & & & &
\end{array}\right), \\
B_{\mu \nu}= & \left(\begin{array}{ccccc}
0 & 0 & 0 & 0 & 0 \\
0 & 0 & x_{1} & 0 & \\
0 & -x_{1} & 0 & & \\
0 & 0 & & 0 \\
0 & &
\end{array}\right),
\end{aligned}
$$

the inverse metric is

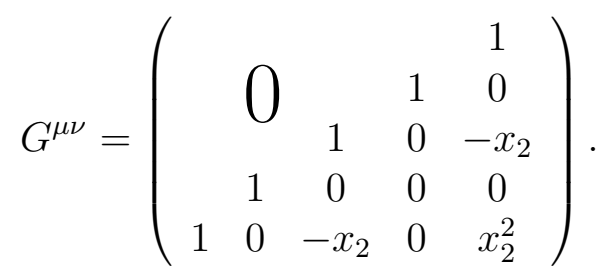

the only non-vanishing component of the Riemann tensor is (up to symmetry) $R_{1212}=\frac{1}{4}$, and the Ricci tensor vanishes.

The torsion of the anti-symmetric tensor is

$$
H=6 d x_{1} \wedge x_{2} \wedge x_{3}
$$

(which means $H_{123}=1$ ) and it is covariantly constant. $H_{\mu \nu}^{2}$ vanishes.

\section{B.7 $\mathcal{A}_{6}$ gauged by $\operatorname{sp}\left\{T_{6}, T_{m}\right\}$}

(eq. (4.47))

The coordinates of the target manifold are

$$
\left\{x_{1}, x_{3}, x_{4}, x_{5}\right\} ;
$$

The background fields are:

$$
G_{\mu \nu}=\left(\begin{array}{cccc}
0 & 2 p x_{1} x_{3}+x_{2} & -2 p x_{3} & 1 \\
2 p x_{1} x_{3}+x_{2} & 1 & -2 p x_{1} & 0 \\
-2 p x_{3} & -2 p x_{1} & 4 p & 0 \\
1 & 0 & 0 & 0
\end{array}\right)
$$




$$
\begin{gathered}
B_{\mu \nu}=2 p\left(\begin{array}{cccc}
0 & -x_{1} x_{3} & x_{3} & 0 \\
x_{1} x_{3} & 0 & -x_{1} & 0 \\
-x_{3} & x_{1} & 0 & 0 \\
0 & 0 & 0 & 0
\end{array}\right) \\
\Phi=-\frac{1}{2} \log \left(x_{1}^{2}+a\right)+\text { const. }
\end{gathered}
$$

where

$$
p=\frac{1}{x_{1}^{2}+a}
$$

the metric has determinant $\operatorname{det} G_{\mu \nu}=-4 a p^{2}$ and signature $(+,+, \operatorname{sign}(a),-)$, so again the signature of the gauged model is obtained (when the gauging is non-singular) by "subtracting" the signature of $d H$ from that of $d G$.

The inverse metric is

$$
G^{\mu \nu}=\frac{1}{a}\left(\begin{array}{cccc}
0 & 0 & 0 & a \\
0 & q_{+} & \frac{1}{2} q_{+} x_{1} & -\left(x_{1} x_{3}+q_{+} x_{2}\right) \\
0 & \frac{1}{2} q_{+} x_{1} & \frac{1}{4} q_{+}^{2} & -\frac{1}{2}\left(q_{-} x_{3}+q_{+} x_{1} x_{2}\right) \\
a & -\left(x_{1} x_{3}+q_{+} x_{2}\right) & -\frac{1}{2}\left(q_{-} x_{3}+q_{+} x_{1} x_{2}\right) & \left(x_{3}^{2}+2 x_{1} x_{2} x_{3}+q_{+} x_{2}^{2}\right)
\end{array}\right)
$$

with $q_{ \pm}=x_{1}^{2} \pm a$;

the non-vanishing components of the Riemann tensor are (up to symmetry)

$$
\begin{aligned}
& R_{1313}=-p^{2}\left(2 x_{1}^{2}-3 a\right) \\
& R_{1314}=2 p^{3} x_{1}\left(2 x_{1}^{2}-a\right) \\
& R_{1414}=-4 p^{3}\left(2 x_{1}^{2}-a\right)
\end{aligned}
$$

the only non-vanishing component of the Ricci tensor is

$$
R_{11}=-2 p^{2}\left(x_{1}^{2}-2 a\right)
$$

and the Ricci scalar vanishes. The torsion of the anti-symmetric tensor is

$$
H=-24 a p^{2} d x^{1} \wedge d x^{3} \wedge d x^{4}
$$

which means $H_{134}=-4 a p^{2}$; The only non-vanishing component of $H_{\mu \nu}^{2}$ is $H_{11}^{2}=8 a p^{2}$ and $H^{2}$ vanishes.

\section{B.8 $\mathcal{A}_{6}$ gauged by $\operatorname{sp}\left\{T_{6}, T_{2}\right\}$}

(eq. (4.53))

The coordinates of the target manifold are

$$
\left\{x_{1}, x_{4}, x_{5}\right\}
$$


the background fields are

$$
\begin{gathered}
G_{\mu \nu}=\left(\begin{array}{ccc}
0 & 0 & 1 \\
0 & \frac{4}{x_{1}^{2}} & 0 \\
1 & 0 & 0
\end{array}\right), \\
B_{\mu \nu}=0 \quad, \quad \Phi=-\log \left(x_{1}\right)+\text { const.; }
\end{gathered}
$$

the inverse metric is

$$
G^{\mu \nu}=\left(\begin{array}{ccc}
0 & 0 & 1 \\
0 & \frac{x_{1}^{2}}{4} & 0 \\
1 & 0 & 0
\end{array}\right) ;
$$

the only non-vanishing component of the Riemann tensor is (up to symmetry) $R_{1441}=\frac{8}{x_{1}^{4}}$,

the only non-vanishing component of the Ricci tensor is $R_{11}=-\frac{2}{x_{1}^{2}}$ and the Ricci scalar vanishes.

\section{References}

[1] E. Witten, Non-Abelian bosonisation in two dimensions, Commun. Math. Phys. 92 (1984) 455.

[2] W. Nahm, Gauging symmetries of two-dimensional conformally invariant models, Davis preprint UCD-88-02 (1988);

K. Bardakci, E. Rabinovici and B. Säring, String models with $c<1$ components, Nucl. Phys. B299 (1988) 151;

K. Gawędzki and A. Kupiainen, Coset construction from functional integrals Nucl. Phys. B320 (1989) 625;

D. Karabali, Q-H. Park, H.J. Schnitzer and Z. Yang, A GKO construction based on a path integral formulation of gauged Wess-Zumino-Witten actions, Phys. Lett. B216 (1989) 307;

[3] C.R. Nappi and E. Witten, A Wess-Zumino-Witten model based on a non-semisimple group, hep-th/9310112, Phys. Rev. Lett. 71 (1993) 3751.

[4] E. Kiritsis and C. Kounnas, String propagation in gravitational wave backgrounds, hep-th-9310202, Phys. Lett. B320 (1994) 264.

[5] D.I. Olive, E. Rabinovici and A. Schwimmer, A class of string backgrounds as a semiclassical limit of WZW models, hep-th/9311081, Phys. Lett. B321 (1994) 361.

[6] K. Sfetsos, Gauging a non-semi-simple WZW model, hep-th/9311010, Phys. Lett. B324 (1994) 335. Exact string backgrounds from WZW models based on non-semisimple groups, hep-th/9311093, Int. J. Mod. Phys. A9 (1994) 4759; 
[7] Gauged WZW models and non-Abelian duality, hep-th/9402031, Phys. Rev. D50 (1994) 2784.

[8] A.A Kehagias and P.A.A. Meessen, Exact string background from a WZW model based on the Heisenberg group, hep-th/9403041, Phys. Lett. B331 (1994) 77.

[9] I. Antoniadis and N. Obers, Plane gravitational waves in string theory, hepth/9403191, Nucl. Phys. B423 (1994) 639.

[10] E. Kiritsis, C. Kounnas and D. Lüst, Superstring gravitational wave backgrounds with spacetime supersymmetry, hep-th/9404114, Phys. Lett. B331 (1994) 321.

[11] N. Mohammedi, On bosonic and supersymmetric current algebras for non-semisimple Lie groups, hep-th/9312182, Phys. Lett. B325 (1994) 371.

[12] A.A. Kehagias, All WZW models in $D \leq 5$, hep-th/9406136.

[13] A.A. Tseytlin, On gauge theories for non-semi-simple groups, hep-th/9505129.

[14] J.M. Figueroa-O'Farrill and S. Stanciu, Non-reductive WZW models and their CFTs, hep-th/9506151; On the structure of symmetric self-dual Lie algebras, hepth/9506152.

[15] J.M. Figueroa-O'Farrill and S. Stanciu Non-semi-simple Sugawara constructions, hep-th/9402035, Phys. Lett. B327 (1994) 40.

[16] E. Inonu and E.P. Wigner, On the contraction of groups and their representations, Proc. Natl. Acad. Sci. U.S. 39 (1953) 510.

[17] A. Medina and Ph. Revoy, Algèbres de Lie et produit scalaire invariant, Ann. Scient. Éc. Norm. Sup. 18 (1985) 553.

[18] O. Pelc, in preparation.

[19] C. Klimčík and A.A. Tseytlin, Exact four-dimensional string solutions and Toda-like sigma models from 'null-gauged' WZNW theories, hep-th/9402120, Nucl. Phys. B424 (1994) 71.

[20] C.G. Callan, D. Friedan, E.J. Martinec and M.J. Perry, Strings in background fields, Nucl. Phys. B262 (1985) 593.

[21] K. Bardakci, M. Crescimanno and E. Rabinovici, Parafermions from coset models, Nucl. Phys. B344 (1990) 344;

I. Bars and D. Nemeschansky, String propagation in backgrounds with curved spacetime, Nucl. Phys. B348 (1991) 89;

E. Witten, String theory and black holes, Phys. Rev. D44 (1991) 314. 
[22] T.H. Buscher, A symmetry of the string background field equations, Phys. Lett. B194 (1987) 59; Path-integral derivation of quantum duality in non-linear sigma-models, Phys. Lett. B201 (1988) 466.

[23] A. Giveon, and M. Roček, On non-Abelian duality, hep-th/9308154, Nucl. Phys. B421 (1994) 173;

E. Alvarez, L. Alvarez-Gaumé and Y. Lozano, On non-Abelian duality, hepth/9403155, Nucl. Phys. B424 (1994) 155;

S. Elitzur, A. Giveon, E. Rabinovici, A. Schwimmer and G. Veneziano, Remarks on non-Abelian duality, hep-th/9409011, Nucl. Phys. B435 (1995) 147.

[24] For a review, see: A. Giveon, M. Porrati, and E. Rabinovici, Target space duality in string theory, hep-th/9401139, Phys. Rep. 244 (1994) 77.

[25] M. Hausner and J.T. Schwartz, Lie groups and their Lie algebras, Gordon and Breach 1968.

[26] C. Klimčík and A.A. Tseytlin, Duality invariant class of exact string backgrounds, hep-th/9311012, Phys. Lett. B323 (1994) 305;

K. Sfetsos and A.A. Tseytlin, Four-dimensional plane wave string solutions with coset CFT description, hep-th/9404063, Nucl. Phys. B427 (1994) 245. 